\title{
Experimental assay of a fitness landscape on a macroevolutionary scale
}

\author{
Victoria O. Pokusaeva ${ }^{1+}$, Dinara R. Usmanova ${ }^{2+}$, Ekaterina V. Putintseva ${ }^{1+}$, Lorena Espinar ${ }^{3,4}$, \\ Karen S. Sarkisyan ${ }^{1,5}$, Alexander S. Mishin ${ }^{5}$, Natalya S. Bogatyreva ${ }^{3,4,6}$, Dmitry N. Ivankov ${ }^{1,6}$, \\ Arseniy V. Akopyan ${ }^{1}$, Sergey Ya. Avvakumov ${ }^{1}$, Inna S. Povolotskaya ${ }^{4}$, Guillaume J. Filion ${ }^{3,4}$, \\ Lucas B. Carey ${ }^{4 *}$, Fyodor A. Kondrashov ${ }^{1 *}$ \\ ${ }^{1}$ Institute of Science and Technology Austria, Am Campus 1, 3400 Klosterneuburg, Austria. \\ ${ }^{2}$ Department of Systems Biology, Columbia University, New York, 10032, NY, USA. \\ ${ }^{3}$ Bioinformatics and Genomics Programme, Centre for Genomic Regulation (CRG), 88 Dr. \\ Aiguader, 08003 Barcelona, Spain. \\ ${ }^{4}$ Universitat Pompeu Fabra (UPF), 08003 Barcelona, Spain. \\ ${ }^{5}$ Shemyakin-Ovchinnikov Institute of Bioorganic Chemistry, Miklukho-Maklaya 16/10, 117997 \\ Moscow, Russia. \\ ${ }^{6}$ Laboratory of Protein Physics, Institute of Protein Research of the Russian Academy of \\ Sciences, Pushchino, Moscow region, Russia \\ ${ }^{+}$Equal contribution \\ *Authors for correspondence
}

Characterizing the fitness landscape, a representation of fitness for a large set of genotypes, is key to understanding how genetic information is interpreted to create functional organisms. Here we determined the evolutionarily-relevant segment of the fitness landscape of His3, a gene coding for an enzyme in the histidine synthesis pathway, focusing on combinations of amino acid states found at orthologous sites of extant species. Just $15 \%$ of amino acids found in yeast His3 orthologues were always neutral while the impact on fitness of the remaining $85 \%$ depended on the genetic background. Furthermore, at $67 \%$ of sites, substitutions are under sign epistasis, having both strongly positive and negative effect in different genetic backgrounds. $46 \%$ of sites were under reciprocal sign epistasis. Sign epistasis affected few genotypes but involved interaction of multiple sites, shaping a rugged fitness landscape in which many of the shortest paths between highly fit genotypes are inaccessible.

Predicting function and fitness of organisms from their genotypes is the ultimate goal of many fields in biology, from medical genetics to systems biology to the study of evolution ${ }^{1-5}$. Among the conceptual frameworks for understanding the genotype to phenotype connection is the fitness landscape, which assigns a fitness (phenotype) to every possible genotype (sequence) of a gene or genome under consideration ${ }^{4,6}$. The recognition of the importance of the fitness landscape stimulated the development of a variety of theoretical approaches to its description, including its general shape and epistatic interactions between alleles, a key property which determines the complexity of the fitness landscape (see [ref. 4] and references within). Before the advent of next-generation sequencing, experimental assays of the fitness landscape were few and could not address the issue at the sequence level. Recently, large-scale experimental assays described the shape of the fitness landscape a few mutations away from a local fitness peak (see [ref. 7-10] and references within). Also, some assays involving a smaller number of genotypes considered combinations of mutations with established functional ${ }^{11-16}$ or evolutionary ${ }^{17-22}$ significance.

Empirical evidence of the nature of large-scale fitness landscapes mostly comes from the study of genotypes incorporating random mutations ${ }^{4,7-10}$, the majority of which are deleterious ${ }^{7-}$ 10,23 . Thus, our present knowledge of fitness landscapes is primarily driven by the study of deleterious mutations and their interactions, although local adaptive trajectories have also been considered $^{2,4,16,24-26}$. Deleterious mutations were found to engage in synergistic epistasis, 
whereby the joint effect of multiple mutations was stronger than the sum of their individual effects $^{4,7-10,16}$. Furthermore, sign epistasis among random mutations was mostly rare ${ }^{5,7-10,16,27}$, although some of these conclusions differ from study to study (e.g. see $[4,27])$.

Unfortunately, there are fundamental limitations to assaying the fitness landscape on a large or macroevolutionary level with random mutation libraries. The number of genotypes underlying the fitness landscape is the combinatorial set of all amino acids across the length of the protein ${ }^{4,6}$. For example, for the 220 amino acid protein coded by the His 3 gene in Saccharomyces cerevisiae, the fitness landscape is a 220 dimensional genotype space with $20^{220}$ different possible sequences. Such immense spaces are both computationally and experimentally intractable. Fortunately, it may not be necessary to survey all genotypes to study the evolutionary-relevant section of the fitness landscape. Because the vast majority of mutations in protein sequences are deleterious $^{23}$, a randomly sampled protein sequence is non-functional ${ }^{28,29}$.

Here we propose an evolutionary approach for assaying fitness landscapes on a macroevolutionary scale in a high-throughput manner that avoids the random sampling of mostly non-functional sequences. The functionally and evolutionarily relevant section of the fitness landscape can be represented by the combination of extant amino acid states, those found in extant species. This approach, applied previously on a limited scale ${ }^{17-22}$ mitigates the problem of exploring a prohibitively large fitness landscape while highlighting the relationships between evolutionarily-relevant genotypes (Fig. 1a). Crucially, substitutions that have been fixed in evolution are fundamentally different from random mutations, the former are either neutral or beneficial in at least some genetic contexts and represent the driving force of molecular evolution, while the latter are mostly deleterious and are primarily relevant on a microevolutionary scale. Therefore, current empirical data do not shed much light on the impact of interactions between substitutions that fixed in the course of evolution by natural selection. Combinations of extant amino acid states allow one to assay a much wider functionally relevant area of the sequence space than approaches based on random mutagenesis of a single sequence (Fig. 1b,c).

\section{Estimating fitness of evolutionary-relevant genotypes}

We studied His3, a gene coding for imidazoleglycerol-phosphate dehydratase (IGPD, His3p), an enzyme essential for histidine synthesis. In a multiple alignment of His3 orthologues from 21 yeast species we identified 686 extant amino acid states (Supplementary Information 1), which were evenly distributed across the His3p structure (Fig. 1d). These 686 substitutions, which occurred over the course of $\sim 400$ million years of evolution ${ }^{30}$ (Fig. 1b,c) correspond to $\sim 10^{83}$ sequences, even a tiny fraction of which would be too many to survey. Thus, we sectioned His 3 into 12 independent segments such that the full combinatorial set of substitutions that have occurred in His3 during yeast evolution comprised 10,000-100,000 genotypes per segment (see Methods and Supplementary Fig. 1a). The 12 segments were of similar length, constrained by the molecular methods employed for library construction (see Methods), and covered a diverse range of secondary structures and functional elements (Supplementary Fig. 1c). For each of the 12 segments of His3 we performed an independent experiment surveying its fitness landscape. For each segment we used degenerate oligonucleotides to construct genotypes consisting of combinations of amino acids present in extant His3 sequences, and determined the fitness conferred by these genotypes by expressing them in a $\Delta$ his 3 strain of $S$. cerevisiae and measuring the rate of growth (Supplementary Fig. 1b). This way we assayed the fitness landscape of the genotype space that was traversed over the course of the last $\sim 400$ million years of evolution ${ }^{30}$.

Across 11 experiments we measured fitness for a total of 4,018,105 genotypes $(875,151$ unique amino acid sequences) with high accuracy. Of these, 422,717 consist solely of 
102 combinations of extant amino acid states from His3 orthologues, while the remaining genotypes

103

104

105

106

107

108

109

110

111

112

113

114

115

116

117

118

119

120

121

122

123

124

125

126

127

128

129

130

131

132

133

134

135

136

137

138

139

140

141

142

143

144

145

146

147

148

149

150

151

152

153 incorporate other amino acid substitutions (Supplementary Table 1 and Methods). For one segment, 9, the accuracy of our experiment was low, and it was not used in cumulative analyses. For each segment we measured fitness for $60 \%-99.8 \%$ of all possible genotypes from the combinatorial set of selected extant amino acid states found in 21 yeast species and a smaller fraction of combinations found across all domains of life (Supplementary Table 1), characterizing the evolutionary relevant fitness landscape (Fig. 1b). For segment 3 for instance, 11 out of 17 amino acid sites had more than one extant amino acid state: L145=2, L147=2, $\mathrm{Q} 148=3, \mathrm{~K} 151=2, \mathrm{~V} 152=2$, D154=3, L164=3, E165=4, A168=2, E169=4, A170=4, with the full yeast combinatorial set consisting of $2 * 2 * 3 * 2 * 2 * 3 * 3 * 4 * 2 * 4 * 4=55,296$ genotypes out of which we determined the fitness for 48,198 , or $87 \%$ of the possible yeast extant states combinations in our library.

A substantial proportion of combinations of extant amino acid states led to genotypes with low fitness (Fig. 2, Fig. 3a,b, Supplementary Fig. 2), an observation that takes into account the false discovery rate in our data (Supplementary Table 1). This observation could be explained by i) some extant amino acids having a universally deleterious effect, ii) some amino acid states exerting a negative effect on fitness because of intergenic interactions with other $S$. cerevisiae genes, or iii) by epistatic interactions between the extant amino acid states within His $3^{31}$. We exclude the possibility that some extant amino acid states had a universally deleterious effect because no extant amino acid states were present only in unfit genetic backgrounds, genotypes conferring a fitness of zero (Fig. 3c). We exclude the possibility that some extant amino acid states disrupt intergenic interactions because the complete His 3 coding sequences from extant species fully complemented a His3 deletion in $S$. cerevisiae (Supplementary Fig. 3c). Thus, the observed genotypes with low fitness can only be explained by epistatic interactions among extant amino acid states within the His 3 gene in the same or different segments. Remarkably, 85\% (330/389) of substitutions between extant amino acid states had substantially different effects on fitness in different backgrounds (Fig. 3d). By contrast, only $15 \%$ of amino acid substitutions that occurred in His 3 evolution are truly neutral, in the sense that they do not exert strong influence on fitness in any genetic background. Three quarters of the universally neutral substitutions were observed in the disordered region of the protein (44/59). Thus, the His3 fitness landscape across the 11 segments with high accuracy was strongly influenced by epistasis on a macroevolutionary scale, i.e. the impact of an extant amino acid state on fitness often depends on the background in which it occurs ${ }^{31-34}$. An epistatic fitness landscape is rugged in the sense that evolving genotypes must avoid fitness valleys that emerge through deleterious combinations of amino acid states that may also be found in fit genotypes $^{18,19,34-36}$. Characterizing the ruggedness and the mechanisms that determine the underlying epistasis becomes the primary challenge in understanding the fitness landscape of His3.

\section{Unidimensional epistasis of the His3 fitness landscape}

The ruggedness of the fitness landscape can be characterized by different measures of complexity of the underlying epistatic interactions. In the simplest case, epistasis may be unidimensional, in the sense that the fitness landscape can be described as a function of an intermediate variable, the fitness potential ${ }^{37-39}$. The fitness landscape is a function from the space of genotypes to fitness. In analogy with a scalar field, we can characterize the ruggedness of this function with standard measures of complexity if genotypes are arranged in a linear space. The simplest case is that of a linear predictor called the fitness potential: $p=\mathrm{c}_{1} x_{1}+\mathrm{c}_{1} x_{2}+\ldots+\mathrm{c}_{\mathrm{n}} x_{n}$, where $\mathrm{c}_{\mathrm{i}}$ is a coefficient and $x_{i}$ is a binary variable that signifies the presence (1) or absence $(0)$ of a given amino acid at a given position. By definition, $e^{p}$ describes a non-epistatic fitness landscape because the effect of every substitution is multiplicative and it depends only on the 
154 associated c. Any other function of $p$ leads to epistasis. If the $f(p)$ function is "simple", meaning that is has a small number of local extremas, such as a bimodal function, the epistasis is called unidimensional ${ }^{39}$. The limitation of simplicity of $f(p)$ is necessary because any function $\mathrm{f}_{0}\left(\mathrm{x}_{1}, \ldots\right.$, $\mathrm{x}_{\mathrm{n}}$ ) can be represented by a function $f^{\prime}(p)$ and choosing appropriate coefficients $\mathrm{c}_{1}, \ldots, \mathrm{c}_{\mathrm{n}}$ in $p$. Thus, a simple $f(p)$ leads to unidimensional epistasis because the entire genotype space can be reduced to a single dimension ${ }^{39}$.

To quantitatively determine how well fitness differences between genotypes can be explained by unidimensional epistasis we used a deep learning approach to estimate the coefficients $\mathrm{c}$ for each allele $x$ in the fitness potential and determine the best unidimensional function of $p$ that best approximated the fitness landscape. We used a dense neural network architecture composed of three layers. Each neuron in the architecture performed a linear transformation of its input and then applied a nonlinear (sigmoid) function. Hence, by using one neuron in the first layer we obtained a linear combination of the contributions of each amino acid state to fitness potential, which was then non-linearly mapped to fitness by the three layers of the neural network architecture (see Methods; Supplementary Fig.4). Ten segments were described by a threshold function in which organismal fitness remains constant with decreasing fitness potential and then is rapidly reduced to lethal after a certain threshold (Fig. 4a). The ability of the cliff-like threshold fitness function ${ }^{40}$ to predict fitness from genotype varied between the His3 segments from near perfect $\left(r^{2}=0.97\right)$ in segment 7 , to relatively poor $\left(r^{2}=0.44\right)$ in segment 5 (Supplementary Fig. 5). Thus, while the fitness landscape of His 3 is approximately unidimensional for some segments, it has a higher degree of complexity for others.

\section{Ruggedness and multidimensional epistasis of the His 3 fitness landscape}

Ruggedness is a general property of fitness landscapes that quantifies the accessible paths of high fitness that connect fit genotype ${ }^{41-43}$. A path between highly fit genotypes is inaccessible when one of the intermediate genotypes has low fitness ${ }^{6,20-22,41,44}$ (e.g. for genotypes AB and ab, the intermediate are $\mathrm{aB}$ and $\mathrm{Ab}$ ). Such instances also manifest in sign epistasis on the fitness landscape, that the same substitution may be beneficial or deleterious when occurring in a different genetic background ${ }^{44,45}$. To quantify the ruggedness of the His 3 fitness landscape we identified instances of sign epistasis: substitutions between extant amino acid states that were strongly beneficial or strongly deleterious (change in fitness of $>0.4$ in absolute value) depending on the background in which they occurred ${ }^{44}$. Some of these instances may be due to miscalled fitness of very few genotypes. Therefore, we considered a pair of extant amino acid states to be under sign epistasis only when sign epistasis was observed in a statistically significant number of different genetic backgrounds (see Methods).

An example of sign epistasis is the substitution C141S in the second segment that had an opposite effect on fitness depending on amino acid at site 143 (I, V or T). The substitution I143T in turn exhibits sign epistasis depending on the amino acid at site 163 (F, I, V or L) (Fig. 5a). These epistatic interactions can be represented by a graph in which nodes represent a pair of extant amino acid states at a specific site and nodes are connected by edges if strong sign epistasis has been detected between them (C141S - I143T - I163F) (Fig. 4b). We found that 86 out of $128(67 \%)$ sites in our library exhibit sign epistasis and 46\% (59/128) exhibit reciprocal sign epistasis. Most sites showed a sign epistatic interaction with multiple other sites (Fig. 5c,d) demonstrating that, although sign epistasis affects few genotypes, it leads to a fitness landscape that requires the interaction of multiple sites for proper characterization.

The complexity of interaction of sites can be estimated by using the graph of sign epistasis where vertices represent a substitution and edges connect vertices with sign epistasis between them. If only few substitutions display sign epistasis then such a graph would signify 
206

207

208

209

210

211

212

213

214

215

216

217

218

219

220

221

222

223

224

225

226

227

228

229

230

231

232

233

234

235

236

237

238

239

240

241

242

243

244

245

246

247

248

249

250

251

252

253

254

255

256

257

that the fitness landscape is relatively smooth, alternatively, a highly-interconnected graph of such interactions signifies a more rugged landscape $e^{4,5,41-43}$. To measure the relative fitness complexity, we used the maximum clique size of a graph, which approximates the maximum number of simultaneously interacting substitutions. In our data, this measure ranged from two to seven depending on the segment (Supplementary Fig. 6). The ruggedness of the fitness landscape of His 3 is high for most segments, such as segment 5, where it is necessary to consider the simultaneous interaction of at least seven sites to accurately predict the fitness of genotypes consisting of extant amino acid states at these sites ${ }^{38,40}$.

Sign epistasis can appear when fitness is described by a unidimensional function of the fitness potential, for example, when the fitness landscape is a unimodal function with an optimum in an intermediate range of the fitness potential ${ }^{41,45}$. However, sign epistasis may also be a sign of multidimensional epistasis, when a unidimensional function of the fitness potential cannot fully describe genotype fitness ${ }^{39}$. Many genotypes were predicted poorly by a unidimensional function of the fitness potential (Supplementary Fig. 5a). Two lines of evidence suggest that such genotypes reveal the presence of multidimensional epistasis. First, genotypes with a higher number of substitutions influenced by sign epistasis were less well-predicted by a unidimensional fitness function (Fig. 4c and Supplementary Fig. 7b). Second, we explain a larger fraction of genotypes by using a more complex neural network architecture accommodating multiple fitness potentials instead of one. We found that increasing the amount of neurons in the first layer of the neural network architecture, which is equivalent to increasing the number of independent fitness potentials, gradually improves the prediction power of the obtained models for most of the segments (Fig. 4d). Thus, adding dimensions to the function of fitness potential increases the prediction power of the model. For example, for a twodimensional case fitness was described by $f_{l}\left(p_{1}, p_{2}\right)$ with $p_{1}=\mathrm{a}_{1} x_{1}+\mathrm{a}_{1} x_{2} \ldots \mathrm{a}_{\mathrm{n}} x_{n}$ and $p_{2}=\mathrm{b}_{1} x_{1}+$ $\mathrm{b}_{1} x_{2} \ldots \mathrm{b}_{\mathrm{n}} x_{n}$. For several His3 segments, a fitness function with multiple underlying fitness potentials described the fitness landscape more accurately than a simple unidimensional function of a single fitness potential (Supplementary Fig. 7a). For instance, for these segments, fitness function of two fitness potentials described the shape with a higher degree of accuracy than a function of a single fitness potential (Fig. 4d,e). By contrast, epistasis in segment 7 is entirely unidimensional (Fig. 4d,e and Supplementary Information 2); we do not see any improvement in the model's predictive power when adding extra dimensions.

\section{Evolutionary trajectories on the His3 fitness landscape}

On a smooth fitness landscape, evolution can proceed along any of the evolutionary paths connecting two fit genotypes, as none of the intermediate genotypes confer low fitness (see Box 2 in [46]). Alternatively, the fitness landscape is rugged when it contains non-connected fitness peaks, such that there are no viable paths between some pairs of genotypes that confer high fitness $^{4,5}$. In other words, the presence of deleterious intermediate genotypes between highly fit ones leads to inaccessibility of some evolutionary trajectories between extant or ancestral sequences ${ }^{6,20-22,44}$. The simplest explanation for the substantial ruggedness of the landscape observed in many of the His 3 segments lies in the unidimensional threshold fitness function (Fig. 6a). On a threshold function a combinations of substitutions, all of which are neutral in some genetic backgrounds, can take a genotype beyond the fitness threshold through their additive effect on fitness potential, making some genotypes inaccessible for evolution (Fig. 6a). Between any two fit genotypes, the fraction of intermediate genotypes that are unfit depends on the fitness potential of the two parental genotypes (Fig. 6b). Evolution between two fit genotypes with high fitness potential can proceed unhindered because all intermediate genotypes also have high fitness potential and, consequently, high fitness. Conversely, when both fit genotypes are located close to the threshold, many of the intermediate genotypes between them have low fitness and many evolutionary paths between them are inaccessible (Fig. 6c). Thus, the 
258

cliff-like threshold fitness function is the major determinant of the observation that not all paths between two fit genotypes are accessible to evolution (Fig. 6b). We find that unfit intermediate genotypes are in genetic proximity with each other and are on a limited number of paths; the fraction of inaccessible paths is smaller than if the same number of unfit genotypes were distributed randomly in genotype space (Fig. 6d,e).

The effect of synergistic epistasis dominates the His3 fitness landscape, affecting over $85 \%$ of amino acid substitutions from our library that occurred in His 3 evolution. This synergistic epistasis may reflect the free energy of the protein ${ }^{10,47,48}$, as evidenced by a correlation between the fitness potential and the impact of substitutions on the free energy of His3p (Supplementary Fig. 8). Similarly, instances of sign epistasis may also be explained by changes in protein stability; for example, in the 143T background C141S increased fitness and also had a positive effect on stability (Fig. 5b). Consistent with protein stability contributing to the observed sign epistasis we find that sites that exhibited reciprocal sign epistasis are close together in the His3p structure (Supplementary Fig. 8). However, an additive contribution to free energy can lead only to a unidimensional fitness function ${ }^{47}$, indicating that other nonadditive mechanisms, such as catalytic activity or inter-subunit interactions, or a non-additive model of free energy, must be responsible for the multidimensionality of the His 3 fitness landscape.

\section{Inference of inter-segmental epistatic interactions in the His3 gene sequence}

Epistasis may be caused by interaction among positions within a segment (intrasegmental epistasis) or by interaction of the segment with the rest of the S. cerevisiae His 3 sequence (inter-segmental epistasis). The contribution of inter- versus intra-segmental interactions can be decoupled. Given two fit genotypes (e.g. ABC \& abc in one His 3 segment), any unfit intermediate states (e.g. aBc in the same His3 segment) must be due to intra-segmental epistasis because the rest of the protein remains constant. For each segment we took as a measurement of intra-segmental epistasis all pairs of fit genotypes and calculated the proportion of unfit intermediate genotypes as a function of the Hamming distance between the two fit genotypes. We then compared this proportion with the total proportion of all unfit genotypes as a function of Hamming distance from $S$. cerevisiae, a measurement that includes both inter- and intra-segmental epistasis. We found three times more inter-segmental than intra-segmental epistasis (Supplementary Fig. 9), likely because a single segment provides a much smaller target space for interactions than the entire His3 protein. The proportion of sites under epistatic interactions increased exponentially with Hamming distance (Supplementary Fig. 9), analogous to Orr's snowball, the accumulation of genetic incompatibilities in the course of speciation ${ }^{31,49}$.

\section{Conclusions}

The concept of the fitness landscape introduced by Sewall Wright (Figures 1 and 2 in [6]) is an indispensable tool for understanding multiple biological phenomena ${ }^{1-5}$. Experimental highthroughput assays of random mutations have begun to unravel some local properties of fitness landscapes ${ }^{4}$. Here, we described a fitness landscape on a macroevolutionary scale by focusing on amino acid states that have been put through the sieve of natural selection. We found that only $15 \%$ of substitutions that fixed in the evolution of His 3 are universally neutral. For the remaining $85 \%$, substitutions from His3 evolution had a profound influence on each other's effect on fitness, providing an experimental confirmation that epistasis is one of the defining features of molecular evolution ${ }^{33}$. Substitutions that occur in evolution have properties vastly different from those of random mutations, which are mostly deleterious ${ }^{23}$. Therefore, the way in which combinations of extant amino acid states affect fitness may also be different from that of combinations of random mutations. Unexpectedly, we found that the interaction of extant amino 
310 acid states was dominated by synergistic epistasis in a manner similar to that previously found 311 for random mutations ${ }^{7-10,16}$. However, the accumulation of random mutations leads to low fitness 312 much faster than the accumulation of extant amino acid states (compare Figure 2 from [16] and 313 Figure $3 b$ from [10] to Fig. 2a).

The experimental data showing that $85 \%$ of amino acid states found in extant species confer low fitness in a different genetic background lends strong support to the notion that epistasis is a key factor in protein evolution ${ }^{31,33}$. We showed that the fitness landscape of several segments of the His 3 gene cannot be reduced to a single unidimensional forms of epistasis, with a function of multiple fitness potentials providing a more accurate description of the fitness landscape. By contrast, large-scale fitness landscapes incorporating multiple random mutations away from the wildtype sequence in a constant test environment have not displayed evidence of multidimensional epistasis ${ }^{8-10,16}$; however, it appears to be a more prevalent factor among substitutions that have been subject to positive selection ${ }^{12-16,18-22,34-36}$. We also found that up to $67 \%$ of sites with an extant amino acid state were influenced by sign epistasis, resulting in a rugged fitness landscape and a limited number of fitness ridges connecting extant sequences for most His 3 segments. Overall, the evolutionary-relevant section of the His 3 fitness landscape is best described as a fitness ridge, with the crest of the ridge defined by a fitness potential. In some cases, the crest is multidimensional requiring several independent underlying fitness potentials. Evolution can proceed unhindered along the crest (Fig. 6a,c), however, pathway availability declines rapidly when evolution proceeds close to the edge of the fitness ridge.

\section{Acknowledgements}

We thank Ben Lehner for a thorough study of the concept behind our experiments and Julia Domingo for technical assistance with preliminary analysis of our data. Jochen Hecht of the CRG Genomics Unit and Svetlana Belogorodtdeva and Roman Belogorodtsev provided technical assistance. The work was supported by HHMI International Early Career Scientist Program (55007424), the MINECO (BFU2012-31329, BFU2012-37168, BFU2015-68351-P and BFU2015-68723-P), Spanish Ministry of Economy and Competitiveness Centro de Excelencia Severo Ochoa 2013-2017 grant (SEV-2012-0208), the Unidad de Excelencia María de Maeztu funded by the MINECO (MDM-2014-0370), Secretaria d'Universitats i Recerca del Departament d'Economia i Coneixement de la Generalitat AGAUR program (2014 SGR 0974), the CERCA Programme of the Generalitat de Catalunya, Russian Foundation for Basic Research grant (1804-01173), the European Union's Horizon 2020 research and innovation programme under the Marie Skłodowska-Curie programme (665385) and the European Research Council under the European Union's Seventh Framework Programme (FP7/2007-2013, ERC grant agreement 335980_EinME and Synergy Grant 609989).

Author contribution. ISP, LBC and FAK conceived the general approach of the study. FAK, LBC, VOP, LE and GJF participated in detailed experimental design. VOP, DRU and EVP spearheaded the experiment, data analysis and main data interpretation, respectively. LE performed a large fraction of the experimental work. AVA and SYaA participated in working on dimensionality. KSS, ASM, NSB, DNI and GJF participated in data analysis. LBC and FAK wrote the draft.

\section{Methods}

\section{Data access}

The raw and processed data have been submitted to the NCBI Gene Expression Omnibus (GEO;http://www.ncbi.nlm.nih.gov/geo/) under accession number GSE99990. A virtual machine containing a running version of the data processing pipeline is available as a Docker image 
361 https://hub.docker.com/r/guil1aume/epi/. The scripts to reproduce the figures are on Github at

\section{Study design}

The His 3 gene was selected for three principal reasons, it is short, conditionally essential and was not known to be involved in protein-protein interactions. Studying $20^{220}$ variants of His 3 is impossible, thus, we have chosen an approach to survey the fitness landscape in a manner that would elucidate the area most relevant to His3 evolution while managing the technical limitations of our experimental design. We considered amino acid states found in extant species, focusing on yeast species, which translated into a full combinatorial set of $\sim 10^{83}$ unique genotypes. Technically it is feasible to measure fitness of up to 100,000 unique genotypes in a single growth experiment. Therefore, we split the His3 gene into 12 independent segments such that the full combinatorial set of extant amino acid states from 21 yeast species in each segment was $10,000-100,000$ genotypes. We then considered the combinatorial library for each segment in an independent growth experiment, which allowed us to study a tractable section of the sequence space while considering trajectories across a vast part of the space connecting extant species (Fig. 1a). We constructed these combinations in 12 plasmid libraries and transformed them into a haploid His3 knockout $S$. cerevisiae strain. Growth rate (fitness) of yeast carrying different mutations in His 3 was measured using serial batch culture in the absence of histidine.

We split the His3 gene sequence into segments in a manner agnostic to the structure of the His3 protein (Supplementary Fig. 1a). For technical reasons, a segment consisted of two variable regions with a constant region between them (Supplementary Fig. 1b). All growth experiments were performed independently for each segment, with the exception of one experiment on a limited group of genotypes from each segment which was done for the normalization of fitness values across different segments (Supplementary Fig. 3).

As a control, we measured the rate of growth of $S$. cerevisiae whose entire His 3 gene sequence came from another distant species. We found that the replacement of an entire gene sequence of His3 leads to wild-type rates of growth of $S$. cerevisiae even when the His3 sequence comes from very distant yeasts, as far as $S$. pombe (Supplementary Fig. 3). Therefore, His 3 appears to be an independent unit of the fitness landscape and is a good model for the study of fitness landscapes of an isolated gene.

\section{Measuring fitness}

Plasmid construction

The His3 open reading frame of $S$. cerevisiae was PCR amplified with its regulatory region from 622 base pairs (bp) upstream of the open reading frame (ORF) to $237 \mathrm{bp}$ downstream of the ORF, using primers 126 and 127 (see Supplementary Table 1) from the wild-type prototroph strain FY4. The PCR product was cloned into vector pRS416 using Gibson assembly (NEB, E2611S). The His3 orthologues from other species were amplified from genomic DNA using designed primers (Supplementary Table 1) and were cloned into the vector pRS416_his3, replacing the ORF of $S$. cerevisiae by Gibson assembly (NEB, E2611S). Since the His 3 orthologue from A. nidulans contains an intron, the whole open reading frame was initially cloned into the vector, and the intron was later removed by PCR-amplifying the whole plasmid without this sequence, followed by recircularization.

\section{Genomic DNA extraction}

Genomic DNA from fungi (Saccharomyces cerevisiae, Saccharomyces bayanus, Candida glabrata, Saccharomyces castellii, Kluyveromyces lactis, Eremotheciumgossypii, Debaryomyces hansenii, Lodderomycese longosporus, Aspergillus nidulans, Schizosaccharomyces pombe, Candida guilliermondii, Saccharomyces kluyveri, Kluyveromyces waltii) was extracted using MasterPure ${ }^{\mathrm{TM}}$ Yeast DNA Purification Kit according to the manufacturer's instructions (Epicentre, MPY80200). 


\section{Mutant library construction}

Twelve independent mutant libraries, each for different regions of His3 (Supplementary Table 1), were generated based on the results of multiple alignment of His 3 orthologues. The alignment was built using the ClustalW alignment feature of the MEGA 6.0 software package ${ }^{50}$ and user-corrected.

Mutant libraries were constructed by fusion-PCR, leaving two variable regions separated by a constant region. For each library, two contiguous fragments of His3 were amplified independently, using $1 \mu \mathrm{g}$ of $S$. cerevisiae (strain FY4) genomic DNA in separate Phusion polymerase reaction mixes (Thermo Fisher Scientific, F530S) in GC buffer. For each PCR, one of the primers was a degenerate oligonucleotide with a constant part at the 5' end required for the fusion-PCR; the other primer was either 126 or 127 . The degenerate primer approach led to the integration of non-extant amino acid sequences due to the redundancy of the genetic code. Consider the amino acid Phe in S. cerevisiae coded by the codon TTT. When incorporating an extant orthologous state Trp (TGG) two independent $T->$ G nucleotide mutations will be incorporated creating the codons TTG (Leu) and TGT (Cys). If these two amino acids were not found in other species then they would be non-extant. The cycling conditions for the PCR were $98^{\circ} \mathrm{C}$ for $30 \mathrm{~s} ; 98^{\circ} \mathrm{C}$ for $20 \mathrm{~s}, 60^{\circ} \mathrm{C}$ for $30 \mathrm{~s}$ and $72^{\circ} \mathrm{C}$ for $1 \mathrm{~min}(25 \mathrm{cycles})$; and $72^{\circ} \mathrm{C}$ for $5 \mathrm{~min}$. The products were column-purified (QIAGEN, QIAquick PCR purification kit, 28104), eluted in $50 \mu \mathrm{l}$ and mixed in equimolar proportion. The fusion-PCR was carried out by diluting $10 \mu \mathrm{l}$ of the mix to $25 \mu \mathrm{L}$ of standard Phusion polymerase reaction mix in GC buffer. The cycling conditions of the fusion-PCR were $98^{\circ} \mathrm{C}$ for $30 \mathrm{~s} ; 98^{\circ} \mathrm{C}$ for $30 \mathrm{~s}, 60^{\circ} \mathrm{C}$ for $2 \mathrm{~min}$ and $72^{\circ} \mathrm{C}$ for 1 min $\left(25\right.$ cycles); and $72^{\circ} \mathrm{C}$ for $5 \mathrm{~min}$. The product of fusion was purified from agarose gel (Qiagen, MinElute Gel Extraction Kit, 28604) and eluted in $10 \mu 1$ of water. $10 \mu 1$ of the product was used as a template for additional 5 cycles of PCR reaction in Phusion polymerase reaction mix (Thermo Fisher Scientific, F530S) in GC buffer, using primers 126 and 127 . The cycling conditions were as follows: $98^{\circ} \mathrm{C}$ for $30 \mathrm{~s} ; 98^{\circ} \mathrm{C}$ for $20 \mathrm{~s}, 60^{\circ} \mathrm{C}$ for $30 \mathrm{~s}$ and $72^{\circ} \mathrm{C}$ for $1 \mathrm{~min}(5$ cycles); and $72^{\circ} \mathrm{C}$ for $5 \mathrm{~min}$. The product was column-purified (QIAGEN, QIAquick PCR purification kit, 28104), and used as an insert for Gibson assembly.

To create a library of His 3 mutants, pRS416 plasmid was amplified using primers 128 and 129. The insert was cloned into the vector using Gibson assembly (NEB, E2611S). Ligated products $(200-300 \mathrm{ng} / \mu \mathrm{L})$ were desalted by drop dialysis using $13 \mathrm{~mm}$ diameter, Type-VS Millipore membrane (Merck Millipore, VSWP01300). $20 \mu \mathrm{L}$ ElectroMAX DH10B competent cells (Invitrogen, 18290015) were electroporated with $3 \mu \mathrm{L}$ ligated products. $0.01 \%$ of the electroporated bacteria were plated on ampicillin-containing medium in order to estimate the complexity of the library; the remaining culture was grown overnight in $100 \mathrm{ml}$ of liquid medium, and the plasmid was extracted the next day. For each library, the maximum number of protein sequences that can be generated was computed. Libraries were generated until to total complexity reached at least 3 times this value.

\section{Yeast transformation and yeast library generation}

For each segment, yeast strain LBCY47 (his 3:KanMXleu2 $\Delta 0$ met15 10 ura3 $\Delta 0$, derived from BY4741) was transformed with $50 \mu \mathrm{g}$ of pRS416_His3 mutant library using lithium acetate transformation and plated onto glucose synthetic complete dropout plates lacking uracil. After 40 hours' growth at $30^{\circ} \mathrm{C}$, approximately 0.5 million yeast colonies were scraped off the plates, mixed together and washed 2 times with $100 \mathrm{ml}$ of PBS.

\section{Bulk competition}

$4 \times 10^{9}$ cells were inoculated into $500 \mathrm{ml}$ of glucose synthetic complete dropout medium lacking uracil with $200 \mathrm{mg} / \mathrm{L}$ of $\mathrm{G} 418$, and grown at $30^{\circ} \mathrm{C}$ at $220 \mathrm{RPM}$ for $6-8 \mathrm{~h}$ in order to eliminate clones with low fitness irrespective of histidine biosynthesis. Cells were later pelleted and washed with $50 \mathrm{ml}$ of PBS. Approximately $10^{10}$ cells were inoculated into $1 \mathrm{~L}$ of synthetic 
465

466

467

468

469

470

471

472

473

474

475

476

477

478

479

480

481

482

483

484

485

486

487

488

489

490

491

492

493

494

495

496

497

498

499

500

501

502

503

504

505

506

507

508

509

510

511

512

513

514

515

516 complete dropout medium lacking histidine, and grown at $30^{\circ} \mathrm{C}$ at $220 \mathrm{RPM}$ for $168 \mathrm{~h}$ with $12 \mathrm{~h}$ between bottlenecks: $\sim 10^{10}$ cells were transferred into fresh medium $\sim 10^{8}$ cells from the culture were kept as sample for the given time point. Bulk competition for each library of mutants was done in two replicates to account for biological variability.

\section{NGS library preparation}

The relative abundance of yeast mutants was measured in 3 samples: 1) the initial population before selection was applied ( $\mathrm{t} 0), 2$ ) the population after $12 \mathrm{~h}$ of growth in the selective medium (t1), and 3) the final population after $168 \mathrm{~h}$ of growth in the selective medium (t14). In order to extract plasmid DNA, $5 \times 10^{9}$ cells from each sample were incubated in $300 \mu \mathrm{L}$ of zymolyase buffer ( $1 \mathrm{M}$ sorbitol, $0.1 \mathrm{M}$ sodium acetate, 60mM EDTA ( $\mathrm{pH} 7.0), 2 \mathrm{mg} / \mathrm{ml}$ zymolyase, $1 \%$ 2-Mercaptoethanol) at $37^{\circ} \mathrm{C}$ for $3 \mathrm{~h}$. The plasmid DNA was purified from the obtained spheroplasts using QIAprep Spin Miniprep Kit (QIAGEN, 27104) according to the manufacturer's protocol. The obtained DNA was used as a template in a $25 \mu \mathrm{L}$ of Q5 DNA polymerase reaction mix (NEB, M0491S), using staggered primers for demultiplexing in the following cycling conditions: $98^{\circ} \mathrm{C}$ for $30 \mathrm{~s} ; 98^{\circ} \mathrm{C}$ for $10 \mathrm{~s}, 60^{\circ} \mathrm{C}$ for $30 \mathrm{~s}$ and $72^{\circ} \mathrm{C}$ for $30 \mathrm{~s}(18$ cycles); and $72^{\circ} \mathrm{C}$ for 2 min. PCR products were purified using Agencourt AM Pure XP beads (Beckman Coulter, A63880), and eluted in $40 \mu \mathrm{L}$ of TE buffer ( $\mathrm{pH}$ 8.0). DNA extraction and PCR-amplification were repeated twice for every sample to account for the technical variability.

NGS libraries were prepared from $100 \mathrm{ng}$ of the purified DNA amplicons using Ovation Rapid DR System (Nugen, 0319-32) according to manufacturer's instructions. Each library was visualized on a Bioanalyzer (Agilent Technologies) and quantified by qPCR with a Kapa Library Quantification Kit (Kapa Biosystems, KK4835). Twelve samples were pooled together (accounting for two biological replicates, two technical replicates and three time points) at the final concentration of $4 \mathrm{nM}$, and sequenced in the same lane. Samples were sequenced as 125-bp paired-end reads on a HiSeq2500 sequencer (Illumina) with v4 sequencing chemistry.

\section{Yeast growth assay}

Mutant strains were grown overnight in complete dropout medium lacking uracil. The cultures were diluted to $0.05 \mathrm{OD} 600 \mathrm{~nm}$, and grown for $5 \mathrm{~h}$ in the same medium. $6 \mu \mathrm{L}$ of each culture were transferred into 96 -well plates in $125 \mu \mathrm{L}$ of complete dropout medium lacking histidine. Growth of the strains was monitored by measuring OD $600 \mathrm{~nm}$ every $10 \mathrm{~min}$ using Tecan Infinite M1000 PRO microplate reader equipped with an integrated Stacker module.

The growth rate of individual curves was measured as the inverse of the time to grow from $\mathrm{OD}=0.135=\exp (-2)$ to $\mathrm{OD}=0.368=\exp (-1)$. If the curve did not reach 0.368 , the growth was set to 0 . Curves that crossed 0.135 or 0.368 were excluded. The growth rate of a clone was measured as the median of 6 independent growth experiments. We excluded from the analysis clones with discordance between growth in solid and liquid medium, clones that could not be sequenced or that showed evidence of contamination by sequencing, and clones such that the Kullback-Leibler divergence of their read counts compared to all synonymous clones was greater than 0.22 . The later criterion ensured that the selected clones were not outliers compared to other variants encoding the same protein.

\section{Growth rates of isolated strains}

We isolated 197 strains from all segment libraries of extant amino acid combinations (926 strain per segment) and used Sanger sequencing to determine the sequence. For each strain we performed 6 repeats of growth assay and calculated the average growth rate. Fitness values from competition and growth rates are highly correlated $\left(\mathrm{r}=0.82 \mathrm{p}=10^{-48}\right)$. Correlation was significant and greater than 0.6 for all segments except S9, where all selected genotypes appeared to be neutral (Supplementary Fig. 3). 


\section{Initial data filtering}

The individual sequences of the variants were recovered from pair-end reads with the following steps: the constant region between the two variable regions was identified by inexact matching allowing up to $20 \%$ errors using the Seeq library version 1.1.2 (https://github.com/ezorita/seeq). The reads are not oriented because the Illumina sequencing adapters were added by ligation, so the constant regions were searched on both reads. Forward and reverse reads were swapped when a match was found on the reverse read. This ensured that all of the sequences are in the same orientation. For multiplexing purposes, the sample identity was encoded in the left and right primers used to PCR-amplify the variants. To demultiplex the reads, we used inexact matching with the candidate primers, allowing up to $20 \%$ errors. This approach was faster and less error-prone than using FLASH $^{51}$. To merge the reads, the sequence of the reverse reads was reverse complemented and the constant region was searched by inexact matching allowing up to $20 \%$ errors. The position of the constant part in each read indicated how they must be stitched together. In the region of overlap, the consensus sequence was determined by picking the nucleotide with highest quality as indicated in the quality line of the fastq files. If 'N' persisted in the final sequence, the reads were discarded. The PCR primers were trimmed so that all the sequences of the same competition would start and end at the same location.

Reads that did not have the constant region, that could not be oriented or that could not be demultiplexed were discarded. The remaining errors in the reads were corrected by sequence clustering. We used starcode version 1.0 [ref. 52] with default parameters and allowing up to two errors. The corrected reads were translated using the genetic code. Variants encoding the same proteins were not merged; they were kept separate for downstream analyses. A running Docker virtual machine with commented scripts to replay the whole the process is available for download at https://hub.docker.com/r/guil1aume/epi/.

\section{DNA sequence variant frequency calculation and data filtering}

The total number of reads for 12 segments, 3 time points and 4 replicas are shown in Supplementary Table 1. Genotypes frequencies are defined as the number of reads for a given genotype divided by the total number of reads in that replicate. Mean frequency was calculated over 4 replicas to be used in further analysis. However, to eliminate influence of outliers the median was taken instead of mean if absolute difference between mean and median was greater than the median value. Only genotypes present in both technical replicas of both biological replicas with at least ten reads (summed across all time points) in each of them were kept.

\section{Noise estimation}

The major factors causing noise in genotype frequency measurements are sampling errors, PCR amplification errors and genetic drift during the competition. For all of these factors, the amount of error depends on the genotype frequency. Therefore, we estimated measurement errors as the function of genotype frequency.

For a given segment, time point and a pair of biological or technical replicas for each genotype we calculated the mean frequency and the squared difference of frequencies from these two replicas. We sorted genotypes by mean frequency and grouped them such that each bin contains 5000 genotypes. We calculated the average frequency and the average squared difference in each bin. Additionally, squared error for frequency 0 was set equal to $\frac{1}{2}$.

$562\left(\left(\frac{0.5}{N_{i}}\right)^{2}+\left(\frac{0.5}{N_{j}}\right)^{2}\right)$, where $N_{i}$ and $N_{j}$ are total read numbers in replicas $i$ and $j$. Finally, by linear interpolation we obtained dependencies of squared differences as a function of frequency, $s_{i j}^{2}(f)$, where $i$ and $j$ are different replicas.

Using squared differences from pairwise comparison of replicas we can estimate variance of mean frequency over four replicas. Let numerate replicas 1, 2, 3, 4 where 1, 2 are technical 
replicas of the first biological repeat and 3, 4 are the technical replicas of the second biological repeat. Errors coming from the competition (e.g.: genetic drift) are shared for replicas 1, 2 and for replicas 3, 4. Let's call them $\Delta f_{b_{1}}$ and $\Delta f_{b_{2}}$ and their variances $\sigma_{b_{1}}^{2}$ and $\sigma_{b_{2}}^{2}$ respectively. Technical errors of sampling from the population and from PCR are unique for each replica. Let's call them $\Delta f_{t_{i}}, i=1 . .4$ and their variances $\sigma_{t_{i}}^{2}, i=1 . .4$ respectively. All variances are function of frequency and writing $\sigma_{X_{i}}^{2}$ we assume $\sigma_{X_{i}}^{2}(f)$.

In the introduced notations the mean frequency over 4 replicas is:

$$
\begin{gathered}
f=\frac{1}{4} \cdot\left(f_{1}+f_{2}+f_{3}+f_{4}\right)= \\
\frac{1}{4} \cdot\left(\left(f^{*}+\Delta f_{b_{1}}+\Delta f_{t_{1}}\right)+\left(f^{*}+\Delta f_{b_{1}}+\Delta f_{t_{2}}\right)+\left(f^{*}+\Delta f_{b_{2}}+\Delta f_{t_{3}}\right)+\left(f^{*}+\Delta f_{b_{2}}+\Delta f_{t_{4}}\right)\right)= \\
f^{*}+\frac{1}{2} \cdot\left(\Delta f_{b_{1}}+\Delta f_{b_{2}}\right)+\frac{1}{4} \cdot\left(\Delta f_{t_{1}}+\Delta f_{t_{2}}+\Delta f_{t_{3}}+\Delta f_{t_{4}}\right),
\end{gathered}
$$

where $f^{*}$ is the true frequency. Applying basic properties of variance the variance of mean frequency:

$$
\sigma^{2}=\frac{1}{4} \cdot\left(\sigma_{b_{1}}^{2}+\sigma_{b_{2}}^{2}\right)+\frac{1}{16} \cdot\left(\sigma_{t_{1}}^{2}+\sigma_{t_{2}}^{2}+\sigma_{t_{3}}^{2}+\sigma_{t_{4}}^{2}\right)
$$

To estimate $\sigma_{b_{1}}^{2}, \sigma_{b_{2}}^{2}, \sigma_{t_{1}}^{2}, \sigma_{t_{2}}^{2}, \sigma_{t_{3}}^{2}, \sigma_{t_{4}}^{2}$ we used squared differences from pairwise comparison of replicas calculated above $s_{12}^{2}, s_{13}^{2}, s_{14}^{2}, s_{23}^{2}, s_{24}^{2}, s_{34}^{2}$ :

$$
\begin{gathered}
\mathbb{E}\left[s_{12}^{2}\right]=\mathbb{E}\left[\left(\Delta f_{t_{1}}-\Delta f_{t_{2}}\right)^{2}\right]=\sigma_{t_{1}}^{2}+\sigma_{t_{2}}^{2} \\
\mathbb{E}\left[s_{13}^{2}\right]=\mathbb{E}\left[\left(\Delta f_{b_{1}}+\Delta f_{t_{1}}-\Delta f_{b_{2}}-\Delta f_{t_{3}}\right)^{2}\right]=\sigma_{b_{1}}^{2}+\sigma_{t_{1}}^{2}+\sigma_{b_{2}}^{2}+\sigma_{t_{3}}^{2} \\
\mathbb{E}\left[s_{14}^{2}\right]=\mathbb{E}\left[\left(\Delta f_{b_{1}}+\Delta f_{t_{1}}-\Delta f_{b_{2}}-\Delta f_{t_{4}}\right)^{2}\right]=\sigma_{b_{1}}^{2}+\sigma_{t_{1}}^{2}+\sigma_{b_{2}}^{2}+\sigma_{t_{4}}^{2} \\
\mathbb{E}\left[s_{23}^{2}\right]=\mathbb{E}\left[\left(\Delta f_{b_{1}}+\Delta f_{t_{2}}-\Delta f_{b_{2}}-\Delta f_{t_{3}}\right)^{2}\right]=\sigma_{b_{1}}^{2}+\sigma_{t_{2}}^{2}+\sigma_{b_{2}}^{2}+\sigma_{t_{3}}^{2} \\
\mathbb{E}\left[s_{24}^{2}\right]=\mathbb{E}\left[\left(\Delta f_{b_{1}}+\Delta f_{t_{2}}-\Delta f_{b_{2}}-\Delta f_{t_{4}}\right)^{2}\right]=\sigma_{b_{1}}^{2}+\sigma_{t_{2}}^{2}+\sigma_{b_{2}}^{2}+\sigma_{t_{4}}^{2} \\
\mathbb{E}\left[s_{34}^{2}\right]=\mathbb{E}\left[\left(\Delta f_{t_{3}}-\Delta f_{t_{4}}\right)^{2}\right]=\sigma_{t_{3}}^{2}+\sigma_{t_{4}}^{2}
\end{gathered}
$$

Therefore, the variance of mean frequency $f$ can be found as:

$$
\sigma^{2}=\frac{1}{16} \cdot\left(\left(s_{13}^{2}+s_{14}^{2}+s_{23}^{2}+s_{24}^{2}\right)-\left(s_{12}^{2}+s_{34}^{2}\right)\right)
$$

582 Recalling that variance and squared differences are a function of frequency:

$$
\sigma^{2}(f)=\frac{1}{16} \cdot\left(\left(s_{13}^{2}(f)+s_{14}^{2}(f)+s_{23}^{2}(f)+s_{24}^{2}(f)\right)-\left(s_{12}^{2}(f)+s_{34}^{2}(f)\right)\right)
$$

For each segment and time point we calculated the numerical function $\sigma^{2}(f)$. Then for each genotype having mean frequency $f_{x}$ we estimated its variance as $\sigma^{2}\left(f_{x}\right)$

\section{Merging amino acid genotypes}

We merged nucleotide genotypes that corresponded to the same amino acid sequence and summed their frequencies and variances. We filtered out all genotypes $x$ which had any of following patterns:

$590 f_{x}^{t_{0}}=0, f_{x}^{t_{1}}=0, f_{x}^{t_{2}}>0$ or $f_{x}^{t_{0}}=0, f_{x}^{t_{1}}>0, f_{x}^{t_{2}}=0$ or $f_{x}^{t_{0}}>0, f_{x}^{t_{1}}=0, f_{x}^{t_{2}}>0$. Fraction of such genotypes were $<0.5 \%$ for all segments except $\mathrm{S} 9$, for which it was $4.5 \%$

For further analysis, this amino acid dataset was used except when specified.

\section{Fitness estimation}

\section{exponentially}

Number of cells in a pool with particular genotype $x$ after time interval $t$ increases

$$
n_{x}^{t}=n_{x}^{0} \cdot \operatorname{Exp}\left[s_{x} \cdot t\right]
$$

where $s_{x}$ is absolute fitness. Frequency of genotype $x$ as well depends exponentially on absolute fitness with an additional multiplicative factor: 


$$
f_{x}^{t}=\frac{n_{x}^{t}}{N^{t}}=\frac{n_{x}^{0} \cdot \operatorname{Exp}\left[s_{x} \cdot t\right]}{N^{t}}=\frac{f_{x}^{0} \cdot \operatorname{Exp}\left[s_{x} \cdot t\right]}{N^{t} / N^{0}},
$$

601 where $N^{t}$ and $N^{0}$ are total cell numbers in a pool at time points 0 and $t$. Factor $\frac{1}{N^{t} / N^{0}}$ reflects the total growth of population, it changes with time but is the same for all genotypes. Therefore, we can rewrite genotype frequency at time $t$ as:

605 where $s^{0 t}=\frac{1}{t} \cdot \log \left(\frac{N^{t}}{N^{0}}\right)$

In the measured dataset for each genotype $x$ we have 3 measurements of frequency $f_{x}^{t_{0}}$, $f_{x}^{t_{1}}, f_{x}^{t_{2}}$ and their errors $\sigma^{2}\left(f_{x}^{t_{0}}\right), \sigma^{2}\left(f_{x}^{t_{1}}\right), \sigma^{2}\left(f_{x}^{t_{2}}\right)$. To estimate genotype fitness we minimized relative squared errors of exponential fit as function of fitness $s_{x}$ and initial frequency $f_{x}^{0}$ :

$$
\left(s_{x}, f_{x}^{0}\right)=\operatorname{argmin}_{s_{x}, f_{x}^{0}}\left(\frac{\left(f_{x}^{t_{0}}-f_{x}^{0}\right)^{2}}{\sigma^{2}\left(f_{x}^{t_{0}}\right)}+\frac{\left(f_{x}^{t_{1}}-f_{x}^{0} \cdot \operatorname{Exp}\left[\left(s_{x}-s^{01}\right) \cdot t_{1}\right]\right)^{2}}{\sigma^{2}\left(f_{x}^{1}\right)}+\frac{\left(f_{x}^{t_{2}}-f_{x}^{0} \cdot \operatorname{Exp}\left[\left(s_{x}-s^{02}\right) \cdot t_{2}\right]\right.}{\sigma^{2}\left(f_{x}^{2}\right)}\right)
$$

This formula contains four parameters common for all genotypes from one segment: $s^{01}, s^{02}, t_{1}, t_{2}$. Further we will perform additional shifting and scaling of fitness values (see next section), therefore, without loss of generality we could sets ${ }^{01}=0$ and $t_{1}=1$.Ideally, $t_{2} / t_{1}$ should equal 14, however, we noticed that this ratio does not hold for many segments and fitted $k=t_{2} / t_{1}$ from data instead of using value 14 .

To find specific $s^{02}$ and $k$ for each segment we selected genotypes with high frequencies at $t_{0}\left(t_{0}>25 \cdot 10^{-6}\right)$ which corresponds to $\sim 500-1000$ reads per technical replicate. Each segment contains $10^{3}-10^{4}$ genotypes that meet this criterion. We minimized eq. (1) for selected genotypes trying all possible combinations of $\left(s^{02}, k\right)$ from a grid where $s^{02} \epsilon[0,1.2]$ with step 0.01 and $k \epsilon[1,14]$ with step 0.1 and choose $\left(s^{02}, k\right)$ which gives minimal $(*)$.

Finally, given $\left(s^{02}, k\right)$ for each segment we found $s_{x}$ for each genotype. Errors for fitness values, $s_{x}^{\text {std }}$, were estimated as standard error of best-fit parameter.

For genotypes with frequencies pattern $f_{x}^{t_{0}}>0, f_{x}^{t_{1}}=0, f_{x}^{t_{2}}=0$ fit of eq. (1) cannot be obtained. Therefore we defined upper boundary for their fitness value as $s_{x}^{\text {boundary }}=$ $\log \left(\frac{1}{\max \left(N_{1}^{t_{1}}, N_{2}^{t_{1}}, N_{3}^{t_{1}}, N_{4}^{t_{1}}\right)}\right)$, where $N_{i}^{t_{1}}, i=1 . .4$ are total read numbers at time point $t_{1}$ in $i$ replica.

\section{Fitness rescaling}

We scaled fitness such that lethal genotypes have fitness 0 and neutral genotypes have fitness 1 . We assumed that genotypes with a stop codon or frame shift are lethal. Thus, for each segment we linearly rescaled the fitness distribution so that $95 \%$ of genotypes with nonsense mutations have a fitness of 0 and so that the local maximum of the fitness distribution of genotypes with extant amino acids is 1 . The scaling around the local maximum led to the shift of fitness values of less than $+/-0.025$ in each of the 12 segments compared to the measured wildtype strains and did not affect our results (for scale, we called a substitution non-neutral if its effect on fitness was $>0.4$ ). All fitness values which became smaller than 0 were set to 0 .

\section{Quality control and comparison of synonymous sequences}

We used nucleotide synonymous sequences as an internal control. The error rate for a measurement of fitness of an amino acid sequence depends on the number of synonymous sequences, $n$, that were used to estimate it. Therefore, we estimated the false discovery rates 
642

643

644

645

646

647

648

649

650

651

652

653

654

655

656

657

658

659

660

661

662

663

664

665

666

667

668

669

670

671

675

676

677

678

679

680

681

682

683

684

685

686

687

688

689

690

691

692

693

\section{Predicting fitness using deep learning} state. overfitting, while controlling for accuracy. function: using random train-test splits.

$$
F(x)=\sigma\left(\sum_{i=1}^{20} c_{3, i} \sigma\left(c_{2, i} \sigma\left(c_{1}^{T} x+b_{1}\right)+b_{2, i}\right)\right)
$$

synonymous variants we merged random combination of $n$ of its nucleotide genotypes and estimated fitness. We then calculated the difference between this fitness and the fitness of the corresponding amino acid sequence. We classified case as "false unfit" if difference was $<-0.4$ and as "false fit" if difference was $>0.4$. The fraction of such cases gives us false discovery rates for genotypes having $n$ synonymous variants. To get total false discovery rates for each segment we averaged "false unfit" and "false fit" rates for different $n$ with weights equal to the fractions of genotypes in amino acid dataset which have $n$ synonymous variant (Supplementary Table 1). The high correlation between biological replicas (Supplementary Table 6) confirms high accuracy of our high-throughput experiments, with the exception of segment 9.

To predict the unidimensional fitness function based on additive contribution of extant amino acid states we used deep learning, a powerful machine learning technique, capable of constructing virtually any function, even with a simple neural network architecture. To convert amino acid sequences into a binary feature matrix we used one-hot encoding strategy, in which each feature (column in the matrix) indicates the presence or absence of a particular amino acid

To optimise the accuracy/overfitting ratio, we tested over a hundred of different neural network architectures and parameters. As a starting point, we selected a number of complicated architectures, which describe our data, but are prone to overfitting due to a large number of parameters. We then gradually reduced the number of layers and neurons to reduce the

Our final architecture consists of three layers and 22 neurons in total (Supplementary Fig. 4). Each of the neurons performs a linear transformation of the input and subsequently applies a non-linear activation function (a sigmoid) to the result. The output of the first layer, therefore, is a sigmoid of a linear transformation of the feature vector $\left(c_{1}{ }^{T} x\right)$. The second layer decompresses the hidden nonlinear representation into 20 sigmoids, the combination of which is further linearly transformed with the only neuron of the third layer and wrapped into another sigmoid

where $\sigma(t)=\frac{1}{(1+\exp (-t))}, x-$ is the feature vector, $c_{1}-$ the vector of coefficients, corresponding to the first layer, $c_{n, k}$ - coefficient corresponding to the $n$-th layer and the $k$-th neuron, $b_{n}$ - bias corresponding to the $n$-th layer. Crucially, this relatively simple architecture is capable of fitting virtually any function ${ }^{53}$, thus, in contrast to conventional logistic regression, in our approach we select the correct model from a vast variety of functions.

The key idea of our approach is that the number of neurons in the first layer of the neural network determines the number of linear combinations of mutations (or fitness potentials) used in order to predict mutant fitness. In other words, each neuron in the first layer assigns a single unique weight to every amino acid state in the dataset (Supplementary Fig. 4). Multiplication of such weight vectors and binary genotype vectors result in fitness potential. Thus, the number of neurons in the first layer of the architecture basically determines the dimensionality of epistasis we assume. The obtained fitness potentials are then transformed by a nonlinear phase shift function constructed by the 22 neurons of the neural network.

The architecture simplicity avoids overfitting, which was further prevented by using early stopping and keeping $10 \%$ of data as a test set. The loss function that is being optimised in our experiments is not convex, which leads to a high probability of getting stuck in different local minima. To ensure reproducibility, each of our models was constructed ten independent times 
694

695

696

697

698

699

700

701

702

703

704

705

706

707

708

709

710

711

712

713

714

715

716

717

718

719

720

721

722

723

724

725

726

727

728

729

730

731

732

733

734

735

736

737

738

739

740

741

742

743

744

745
Each model was trained for under 100 epochs using mean squared error as the loss function. An unpublished adaptive learning rate method proposed by Geoff Hinton, RMSProp, was used as the optimiser. This algorithm is a version of a mini-batch stochastic gradient descent, utilising the gradient magnitude of the recent gradients in order to normalise the current ones. All the weights were initialised using Xavier normal initialiser ${ }^{54}$.

\section{Paths between pairs of fit genotypes}

For analysis in Fig. 6d, we first choose two fit "parental" genotypes, one randomly chosen genotype (eg: ABE) and the other parental genotype that is either $S$. cerevisiae wildtype genotype (inter-segmental) or another random fit genotype in the data (intra-segmental) (eg: abe). The two genotypes in this example are Hamming Distance 3 apart $(\mathrm{HD}=3)$. We next compute all (HD2-2) intermediate genotypes (eg: $\mathrm{AbC}$, aBc, et cetera) and retain the subset that were experimentally measured. We represent the two parental genotypes and all measured intermediate genotypes as an undirected graph in which each genotype is a vertex. All genotypes one substitution apart are connected by an unweighted edge. The shortest possible path for a given pair of genotypes is of length HD. We find all shortest paths between the two parental genotypes using a breadth-first search. We next remove all vertices (genotypes) that are unfit, and recompute the number of shortest between the two parental genotypes. For example, in Fig. $\mathbf{6 a}$, there are six paths of length three if you take into account all genotypes, but only three paths of length three if you take into account only fit genotypes.

\section{Clustering of unfit genotypes in sequence space}

For the analysis in Fig. 6e, we first represent the two parental genotypes and all measured intermediate genotypes as an undirected graph in which each genotype is a vertex. All genotypes one substitution apart are connected by an unweighted edge. We can then compute the degree (number of genotypes of distance one) for each vertex (genotype). We do so randomly drawing from all measured genotypes and using only unfit genotypes or using the same number but randomly chosen genotypes. For the randomly chosen genotypes, the value is the average over 1000 runs.

\section{Quantifying sign epistasis}

For each substitution (eg: $\mathrm{C}->\mathrm{S}$ at position 141), we considered only those that exhibit a large fitness effect (abs. difference $>0.4$ ) comprising a set of substitutions with large effects. For each substitution we divided the genetic backgrounds into two categories: those in which the substitution caused a $>0.4$ increase in fitness, and those backgrounds in which the substitution caused $>0.4$ decrease in fitness. A single substitution can cause a large increase in fitness in some backgrounds and a large decrease in others due to two possible reasons: sign epistasis or experimental error. To differentiate the two cases, we identified secondary substitutions that significantly alter the ratio of large increases to large decreases in fitness (Fisher's exact test, Bonferroni corrected $\mathrm{p}$-value $<0.05$ ). We only consider a site to be under sign epistasis if there is a second site that alters the frequency of sign epistasis in a statistically significant manner, i.e. more frequently than expected by chance alone.

\section{Structural analysis}

\section{Structure prediction}

An initial model was obtained with the I-TASSER server ${ }^{55}$. The list of top 10 PDB structural templates picked up by the I-TASSER included high-quality crystal structures of imidazoleglycerol-phosphate dehydratases from Arabidopsis thaliana and Cryptococcus neoformans. Coordinates of the top-scoring model $(\mathrm{C}$-score $=0.21$, estimated $\mathrm{TM}$-score $=$ $0.74 \pm 0.11$, estimated RMSD $=5.1 \pm 3.3 \AA$ ) and the predicted normalized B-factor ${ }^{56}$ were used for further analysis. The value of the model quality metric (TM-score $>0.5$ ) indicates a model of 
746 correct topology. The proteins structurally close to the final model (RMSD $0.6-1.7 \AA$ are PDB 747 IDs 4MU0, 4GQU, 1RHY, 5DNL and 2AE8 from Arabidopsis thaliana, Mycobacterium 748 tuberculosis, Cryptococcus neoformans, Pyrococcus furiosus and Staphylococcus aureus.

749 We measured the distribution of distances (in angstroms) between pairs of residues that 750 exhibit strong sign epistasis (Supplementary Table 1, ReallyPositivePair == TRUE), and 751 compared it with the distribution of pairwise distances among residues for which we have 752 sufficient data to be certain that a given pair does not exhibit sign epistasis (Supplementary Table 1, ReallyNegativePair == TRUE).

\section{$\Delta \Delta G$ prediction}

Cartesian_ddg application ${ }^{57}$ from Rosetta version 2017.08.59291 was used for $\Delta \Delta \mathrm{G}$ predictions. Top-scoring I-TASSER model was pre-minimized using the Relax ${ }^{58}$ application in dual-space $^{59}$ with the flags: -relax:dualspace true; -ex1; -ex2; -use_input_sc; -flip_HNQ; no_optH false; -relax:min_typelbfgs_armijo_nonmonotone; -nonideal. The best scoring model from 1000 structures was selected. The effect of up to 4 mutations $(54,500$ genotypes in total) was assessed in Cartesian space with the Talaris_2014 score function, and the -fa_max_dis 9.0 flag. $\Delta \Delta \mathrm{G}$ was estimated as a difference of mean score for 3 independent runs for every mutant and the wild-type score. 


\section{Figures}

Figure 1. Combinatorial approach to the study of fitness landscapes. A fitness landscape is the representation of fitness for all possible genotypes composed of a specific set of loci. a, Following Figure 1 from Sewall Wright ref. [6] consider the genotype space consisting of 5 loci, each with two allele states (lower and uppercase letters). The entire genotype space is 5dimensional consisting of $2^{5}$ genotypes. Given two genotypes found in extant species (abCde and $\mathrm{ABCdE}$ in this example), surveying combinations of extant alleles substantially reduces the dimensionality of the genotype space, concomitantly reducing the number of genotypes to assay. The surveyed area (blue cube) considers all combinations of allele substitutions that have occurred in the course of evolution between the two sequences (red line), avoiding the sampling of combinations with less relevance to the evolutionary trajectory (black lines). b, Given the entire multidimensional genotype space (black circle) our approach considers an multidimensional subspace consisting of the combinatorial set of amino acid states from extant species. The blue line represents the yeast phylogeny and the surrounding blue space represents a multidimensional set of combinations of extant amino acids of the sequence under consideration, one His3 gene segment in our study. By contrast, random mutagenesis studies consider only a local segment of the genotype space surrounding a specific genotype (green circle). c, A multiple alignment of orthologous sequences of His 3 for segment 2 for which we incorporated almost all extant amino acid states from 21 yeast species (blue bars) and 10-100\% extant states from a set of 396 orthologues (grey bars). d, The predicted structure of His $3 p$ with amino acid residues that were substituted in our library.

Figure 2. Visual representations of the fitness landscape. a, The fitness landscape for all assayed genotypes in segment 7. Nodes represent unique amino acid sequences with edges connecting those separated by a single amino acid substitution. Colour saturation represents the minimum fitness of the two connected nodes. b, For segment 7, fitness of ancestral and extant nodes and genotypes one substitution away from the nodes in the background of $S$. cerevisiae gene on the yeast phylogeny (black lines), are shown in colour ranging from grey (lowest fitness) 
800 Figure 3. Fitness distributions. a, The distribution of fitness for genotypes composed of 801 combination of extant amino acid states (green) and non-extant amino acid states (purple) at the same positions. b, The fraction of unfit genotypes per segment among genotypes consisting entirely from extant amino acid states (green) and those incorporating non-extant amino acid states (purple). c, The number of genotypes with high fitness that incorporates specific amino acid states. For each amino acid state, the number of genetic backgrounds that contain that amino acid state and are fit (fitness $>0$ ) are shown. d, The percent of backgrounds in which a specific substitution is neutral (white), beneficial (blue) or deleterious (grey). The region marked in green shows substitutions that never have large effects $(>0.4)$ on fitness. Beneficial and deleterious effects are shown only if the frequency for a given substitution was higher than the false 
Figure 4. Epistasis and the His3 fitness landscape for segments 2, 5 and 7. a, Fitness as a ction of a single fitness potential (black curve, the fitness of individual genotypes is orange).

817 b, A network depiction of sign epistasis between amino acid substitutions. Colour coded sites 818 with reciprocal sign epistasis (black lines) and unidirectional interactions (grey arrows) are 819 shown. c, Genotypes containing substitutions with a higher number of sign epistatic interactions 820 are less likely to be fit by the threshold function of the fitness potential. d, Increasing the number 821 of neurons in the first layers of the neural network, which is equivalent to increasing the number 822 of underlying fitness potentials, leads to more accurate models for segments with detected sign 823 epistasis. Each dot corresponds to an independent optimization of model parameters. e, Fitness as a function of two fitness potentials (black dots, measured fitness is depicted in orange).

Figure 5. Sign epistasis. a, Substitution $C->S$ at site 141 in segment 2 more frequently has a positive effect on fitness in the background of $\mathrm{T}$ at site 143, a negative effect in the background of 143I and is equally likely to be strongly deleterious or strongly beneficial in the background of $143 \mathrm{~V}$. b, Predicted change in free energy following a C141S substitution in all genetic closer than four mutations away from $S$. cerevisiae. c, Distribution of the number of substitutions at each site under sign (yellow) and 832 reciprocal sign (orange) epistasis. Sites with 0 interactions do not exhibit sign epistasis. d, The 833 fraction of genotypes in which the substitution under sign epistasis has the less frequent effect on 834 fitness. 
838 Figure 6. Analysis of evolutionary pathway accessibility. a, A threshold fitness potential 839 function can lead to some paths being inaccessible between two genotypes of high fitness (abe, $840 \mathrm{ABE}$ ) if the joint contribution of several alleles to the fitness potential (abE, aBE) leads to the 841 fitness potential below the threshold. $\mathbf{b}$, The fraction of unfit intermediate genotypes between 842 two fit genotypes as a function of their average fitness potential. c, The grey area represents all 843 genotypes in segment 7 . When two fit genotypes (red dots) have high fitness potential, many 844 paths between them will be accessible because many intermediate genotypes will also have high 845 fitness potential and fitness (blue dots). d, The fraction of accessible shortest paths between two 846 fit genotypes with unfit genotypes from data (orange) or the same number of randomly selected 847 genotypes (grey), shown as a function of Hamming distance between two fit genotypes. Error 848 bars are standard deviation. e, On a graph with genotypes connected by edges if they are one 849 substitution apart we calculate the degree of connectivity (number of edges for each node) for all 850 genotypes (blue), only unfit (fitness $=0$ ) genotypes (orange) and a graph with the same number 851 of nodes as the graph with unfit genotypes but with nodes chosen at random (grey). 


\section{Supplementary Figures}

Supplementary Figure 1. Experimental design. a, The sequence of the His 3 protein from S. cerevisiae was separated into 12 independent segments of similar lengths, such that the full combinatorial set of extant amino acid substitutions was less than 100,000 possible genotypes. These segments represented different combinations of structural elements of the His 3 protein structure. b, For each of the 12 segments from His3, we selected extant amino acid states using a multiple alignment of His 3 orthologues from 396 species, preferentially incorporating states from 21 yeast species, the variability is shown in segment 3 as an example. Mutant degenerate codon libraries were constructed by fusion PCR of two synthesized variable halves of each segment. These high-complexity plasmid libraries were transformed into haploid His3 knockout S. cerevisiae strain. The growth rate of yeast carrying different extant amino acid state combinations in His3 gene was measured using serial batch culture in the absence of histidine with 12 hours between $\sim 100$-fold dilutions. To estimate the fitness of yeast mutants their relative abundance was measured at three points: in the initial population before selection $(\mathrm{t} 0)$, in the population after 12 hours of growth in the selective medium (t1), and in the final population after 168 hours of growth in the selective medium (t14). To assess the fitness of individual mutants the segments from three populations were amplified and sequenced. The relative abundance of each sequence was used as a proxy for abundance of the associated yeast mutant, which in turn determines its fitness. c, Secondary structure of His 3 mapped to the segments in our experiments.

Supplementary Figure 2. Segment-specific fitness distributions for extant and non-extant amino acid states. a, The fitness distribution for each segment for genotypes consisting only of extant amino acid states (green) or that contain one or more non-extant amino acid states (purple) only at positions with a substitution in the extant library. b, The fitness distribution for each segment for genotypes consisting only of extant amino acid states (green) and genotypes with mutations at other positions in that segment (red).

\section{Supplementary Figure 3. Growth rate measurement of isolated strains. a, Comparison of fitness values from the pooled competition assay with growth rates of isolated strains as measured in a microplate reader. Error bars for growth rates show s.e.m. of 6 replicates. b, Pearson correlation coefficients between fitness values from competition and growth rates of isolated strains for each segment. $* *$ signifies $p$-value $<0.005$ (correlation test). c, His $3 p$ orthologues from different species complement a $\Delta$ his3 deletion in $S$. cerevisiae. Growth rates of transformants containing whole HIS3 orthologous genes from other yeast species. Error bars for growth rates show s.e.m. of $\geq 7$ replicates.}

Supplementary Figure 4. Schematic representation of the deep learning approach. Each genotype was encoded as a binary vector $(x)$. During training, each of the substitutions was assigned a coefficient $\left(c_{i}\right)$, comprising a vector of coefficients $(c)$. The multiplication of these two vectors is the fitness potential of the genotype. After going through three layers, each with a sigmoid activation function, the predicted fitness is obtained.

Supplementary Figure 5. The fitness potential of the His3 fitness landscape. a, Fitness potential predicted by the neural network as a function of the measured fitness for all 12 segments. b, The correlation between the fitness predicted by the fitness potential and the measured fitness. c, Training and test $\mathrm{R}^{2}$ for each segment for 20 -fold cross-validation. 
Supplementary Figure 6. Sign epistasis dimensionality graphs for all twelve segments. Each node represents a substitution, with multiple substitutions at the same site having the same colour. Substitutions under reciprocal sign epistasis are indicated by black lines while grey arrows indicate unidirectional sign epistasis. 

increasing the number of underlying fitness potentials, leads to more accurate models for segments with detected sign epistasis. Each dot corresponds to an independent optimization of model parameters. $\mathbf{b}$, Number of sign epistatic interactions of certain substitutions against average model prediction power for mutants including these substitutions.

Supplementary Figure 8. Protein stability and the fitness potential. a, A comparison of correlation coefficients between predicted and measured values across segments. b,c, correlations between the estimated impact of substitutions on free energy $(\Delta \Delta \mathrm{G})$, fitness potential and fitness. $\Delta \Delta \mathrm{G}$ correlates better with fitness potential than with fitness. d, Pairs of sites that exhibit sign (connected by a light edge in Supplementary Figure 6) and those that exhibit reciprocal sign epistasis (connected by a dark edge in Supplementary Figure 6) are closer together in the His $3 p$ structure that randomly chosen non-connected pairs of positions that exhibit sign epistasis.

Supplementary Figure 9. Decoupling inter- and intra-segmental epistasis. a, The fraction of unfit genotypes between $S$. cerevisiae and any other genotype consisting of extant amino acid states with high (blue) or any (red) fitness, and genotypes in the latter but not the former category (black) as a function of the Hamming distance between the two boundary genotypes. Points indicate median, the bars and lines indicate $50 \%$ of the genotypes and genotypes 2.7 sigmas from the mean, respectively. b, The neural network model assigns higher weights to amino acid states that that first occur in His3 orthologues farther from $S$ cerevisiae, indicating the presence of intrasegmental interactions.

\title{
Supplementary Information 1. Multiple alignment of His3 orthologues.
}

\author{
Supplementary Information 2. Multidimensional description of epistasis in His3 \\ segments. Fitness as a function of two fitness potentials (black dots, measured fitness is depicted \\ in orange).
}




\section{References}

1. Dean, A. M. \& Thornton, J. W. Mechanistic approaches to the study of evolution: the functional synthesis. Nature Rev. Genet. 8, 675-688 (2007).

2. Kogenaru, M., de Vos, M. G. J., \& Tans, S. J. Revealing evolutionary pathways by fitness landscape reconstruction. Crit. Rev. Biochem. Mol. Biol. 44, 169-174 (2009).

3.Mackay, T. F. C. Epistasis and quantitative traits: using model organisms to study gene-gene interactions. Nature Rev. Genet. 15, 22-33 (2014).

4. de Visser, J. A. G. M. \& Krug, J. Empirical fitness landscapes and the predictability of evolution. Nature Rev. Genet. 15, 480-490 (2014).

5. de Visser, J. A. G. M., Cooper, T. F., \& Elena, S. F. The causes of epistasis. Proc. Biol. Sci. 278, 3617-3624 (2011).

6. Wright, S. The roles of mutation, inbreeding, crossbreeding and selection in evolution. Proc. Sixth Int. Congr. Genet. 1, 356-366 (1932).

7. Olson, C. A., Wu, N. C. \& Sun, R. A comprehensive biophysical description of pairwise epistasis throughout an entire protein domain. Curr. Biol. 24, 2643-2651 (2014).

8. Puchta, O., Cseke, B., Czaja, H., Tollervey, D., Sanguinetti, G., and Kudla, G. Network of epistatic interactions within a yeast snoRNA. Science. 352, 840-844 (2016).

9. Li, C. Qian, W. Maclean, C. J. \& Zhang, J. The fitness landscape of a tRNA gene. Science 352, 837-840 (2016).

10. Sarkisyan, K.S., et al. Local fitness landscape of the green fluorescent protein. Nature $\mathbf{5 3 3}$, 397-401 (2016.)

11. Hietpas, R. T., Bank, C., Jensen, J. D. \& Bolon, D. N. A. Shifting fitness landscapes in response to altered environments. Evolution 67, 3512-3522 (2013).

12. Parera, M. \& Martinez, M. A. Strong epistatic interactions within a single protein. Mol. Biol. Evol. 31, 1546-1553 (2014).

13. Podgornaia, A. I. \& Laub, M. T. Pervasive degeneracy and epistasis in a protein-protein interface. Science 347, 673-677 (2015).

14. Anderson, D. W. McKeown, A. N. \& Thornton, J. W. Intermolecular epistasis shaped the function and evolution of an ancient transcription factor and its DNA binding sites. Elife 4, e07864 (2015).

15. Meini, M. R., Tomatis, P. E., Weinreich, D. M. \& Vila, A. J. Quantitative description of a protein fitness landscape based on molecular features. Mol. Biol. Evol. 32, 1774-1787 (2015). 
994

995

996

997

998

999

1000

1001

1002

1003

1004

1005

1006

1007

1008

1009

1010

1011

1012

1013

1014

1015

1016

1017

1018

1019

1020

1021

1022

1023

1024

1025

1026

1027

1028

1029

1030

1031

1032

1033

1034

1035

1036

1037

1038

1039

1040

1041

1042

1043

1044
16. Bank, C., Matuszewski, S., Hietpas, R. T. \& Jensen, J. D. On the (un)predictability of a large intragenic fitness landscape. Proc. Natl. Acad. Sci. USA. 113, 14085-14090 (2016).

17. Poelwijk, F.J., Kiviet, D.J., Weinreich, D.M., \& Tans, S.J. Empirical fitness landscapes reveal accessible evolutionary paths. Nature 445, 383-386(2007).

18. Gong, L. I. Suchard, M. A.\& Bloom, J.D. Stability-mediated epistasis constrains the evolution of an influenza protein. Elife 2, e00631(2013).

19. Gong, L. I.\& Bloom, J.D. Epistatically interacting substitutions are enriched during adaptive protein evolution. PLoS Genet. 10, e1004328(2014).

20. Starr, T. N. \& Thornton, J. W. Epistasis in protein evolution. Protein Sci. 25, 1204-1218 (2016).

21. Kumar, A. Natarajan, C. Moriyama, H. Witt, C.C. Weber, R.E. Fago, A.\& Storz, J.F. Stability-mediated epistasis restricts accessible mutational pathways in the functional evolution of avian hemoglobin. Mol. Biol. Evol. 34, 1240-1251(2017).

22. Tufts, D. M., et al. Epistasis constrains mutational pathways of hemoglobin adaptation in high-altitude pikas. Mol. Biol. Evol. 32, 287-298 (2015).

23. Eyre-Walker A. \& Keightley P. D. The distribution of fitness effects of new mutations. Nat. Rev. Genet. 8, 610-618 (2007).

24. Draghi, J. A. \& Plotkin, J. B. Selection biases the prevalence and type of epistasis along adaptive trajectories. Evolution 67, 3120-3131 (2013).

25. Lunzer, M., Miller, S. P., Felsheim, R. \& Dean, A. M. The biochemical architecture of an ancient adaptive landscape. Science 310, 499-501 (2005).

26. Palmer, A. C., Toprak, E., Baym, M., Kim, S., Veres, A., Bershtein, S. \& Kishony, R. Delayed commitment to evolutionary fate in antibiotic resistance fitness landscapes. Nat. Commun. 6, 7385 (2015).

27. Lalić, J. \& Elena, S. F. Magnitude and sign epistasis among deleterious mutations in a positive-sense plant RNA virus. Heredity 109, 71-77 (2012).

28. Keefe, A. D. \& Szostak, J. W. Functional proteins from a random-sequence library. Nature 410, 715-718 (2001).

29. du Plessis, L. Leventhal, G.E. \& Bonhoeffer, S. How good are statistical models at approximating complex fitness landscapes? Mol. Biol. Evol. 33, 2454-2468(2016).

30. Lum, P. Y. Edwards, S.\& Wright, R. Molecular, functional and evolutionary characterization of the gene encoding HMG-CoA reductase in the fission yeast, Schizosaccharomyces pombe. Yeast 12, 1107-1124 (1996).

31. Kondrashov, A.S. Sunyaev, S. \& Kondrashov, F. A. Dobzhansky-Muller incompatibilities in protein evolution. Proc. Natl. Acad. Sci. USA 99, 14878-14883 (2002). 
1045

1046

1047

1048

1049

1050

1051

1052

1053

1054

1055

1056

1057

1058

1059

1060

1061

1062

1063

1064

1065

1066

1067

1068

1069

1070

1071

1072

1073

1074

1075

1076

1077

1078

1079

1080

1081

1082

1083

1084

1085

1086

1087

1088

1089

1090

1091

1092

1093

1094

32. Kvitek, D. J. \& Sherlock, G. Reciprocal sign epistasis between frequently experimentally evolved adaptive mutations causes a rugged fitness landscape. PLoS Genet.7, e1002056 (2011).

33. Breen, M. S., Kemena, C., Vlasov, P. K., Notredame, C. \& Kondrashov, F. A. Epistasis as the primary factor in molecular evolution. Nature 490, 535-538 (2012).

34. Gavrilets, S. Evolution and speciation on holey adaptive landscapes. Trends Ecol. Evol. 12, 307-312(1997).

35. Salverda, M. L. M., Dellus, E., Gorter, F. A., Debets, A.J. M., van der Oost, J. Hoekstra, R. F. Tawfik, D. S. \& de Visser, J.A.G. M. Initial mutations direct alternative pathways of protein evolution. PLoS Genet. 7, e1001321(2011).

36. Weinreich, D. M., Delaney, N. F., de Pristo, M. A. \& Hartl, D.L. Darwinian evolution can follow only very few mutational paths to fitter proteins. Science 312, 111-114(2006).

37. Milkman, R. Selection differentials and selection coefficients. Genetics 88, 391-403 (1978).

38. Kimura, M. \& Crow J. F. Effect of overall phenotypic selection on genetic change at individual loci. Proc. Natl. Acad. Sci. USA. 75, 6168-6171 (1978).

39. Kondrashov, F. A. \& Kondrashov, A. S. Multidimensional epistasis and the disadvantage of sex. Proc. Natl. Acad. Sci. USA. 98, 12089-12092 (2001).

40. Crow, J. F. \& Kimura, M. Efficiency of truncation selection. Proc. Natl. Acad. Sci. USA 76, 396399 (1979).

41. Weinreich, D. M. Lan, Y. Wylie, C. S.\& Heckendorn, R. B. Should evolutionary geneticists worry about higher-order epistasis? Curr. Opin. Genet. Dev. 23, 700-707 (2013).

42. Poelwijk, F.J. Krishna, V. \& Ranganathan, R. The Context-Dependence of Mutations: A Linkage of Formalisms. PLoS Comput. Biol. 12, e1004771 (2016).

43. Sailer, Z. R. \& Harms, M. J. High-order epistasis shapes evolutionary trajectories. PLoS Comput. Biol. 13, e1005541 (2017).

44. Weinreich, D. M. Watson, R. A. \& Chao, L. Perspective: Sign epistasis and genetic constraint on evolutionary trajectories. Evolution 59, 1165-1174 (2005).

45. Rokyta, D. R. et al. Epistasis between beneficial mutations and the phenotype-to-fitness Map for a ssDNA virus. PLoS Genet. 7, e1002075 (2011).

46. Kondrashov, D. A. \& Kondrashov, F. A. Topological features of rugged fitness landscapes in sequence space. Trends Genet. 31, 24-33 (2015).

47. De Pristo, M. A. Weinreich, D. M. \& Hartl, D. L. Missense meanderings in sequence space: a biophysical view of protein evolution. Nature Rev. Genet. 6, 678-687 (2005).

48. Bershtein, S., Segal, M., Bekerman, R., Tokuriki, N. \& Tawfik, D. S. Robustness epistasis link shapes the fitness landscape of a randomly drifting protein. Nature 444, 929-932 (2006).

49. Orr, H. A. The population genetics of speciation: the evolution of hybrid incompatibilities. Genetics 139, 1805-1813 (1995). 
1095 50. Tamura, K., Stecher, G., Peterson, D., Filipski, A. \& Kumar, S. MEGA6: Molecular

1096 Evolutionary Genetics Analysis version 6.0. Mol Biol Evol. 30, 2725-2729 (2013).

1097 51. Magoč, T. \& Salzberg, S. L. FLASH: fast length adjustment of short reads to improve

1098 genome assemblies. Bioinformatics 27, 2957-2963 (2011).

1099 52. Zorita, E., Cuscó, P. \& Filion, G. J. Starcode: sequence clustering based on all-pairs search.

1100 Bioinformatics 31, 1913-1919 (2015).

1101 53. LeCun, Y., Bengio, Y. \& Hinton, G. Deep learning. Nature. 521, 436-444 (2015).

1102 54. Glorot, X. \& Bengio, Y. Understanding the difficulty of training deep feedforward neural 1103 networks. In Proceedings of the 13th International Conference on Artificial Intelligence and 1104 Statistics (2010).

1105

1106

1107

55. Yang, J. \& Zhang, Y. I-TASSER server: new development for protein structure and function

1108 56. Yang, J., Wang, Y. \& Zhang, Y. ResQ: An Approach to Unified Estimation of B-Factor and 1109 Residue-Specific Error in Protein Structure Prediction. J. Mol. Biol. 428, 693-701 (2016).

1110

1111 57. Park, H., Bradley, P., Greisen, P. Jr., Liu, Y., Mulligan, V. K., Kim, D.E., Baker, D. \&

1112 DiMaio, F. Simultaneous optimization of biomolecular energy functions on features from small

1113 molecules and macromolecules. J. Chem. Theory Comput. 12, 6201-6212 (2016).

1114 58. Nivón, L. G., Moretti, R. \& Baker, D. A Pareto-optimal refinement method for protein 1115 design scaffolds. PLoS One 8, e59004 (2013).

1116 59. Conway, P., Tyka, M. D., DiMaio, F., Konerding, D. E. \& Baker, D. Relaxation of backbone 1117 bond geometry improves protein energy landscape modeling. Protein Sci. 23, 47-55 (2014). 


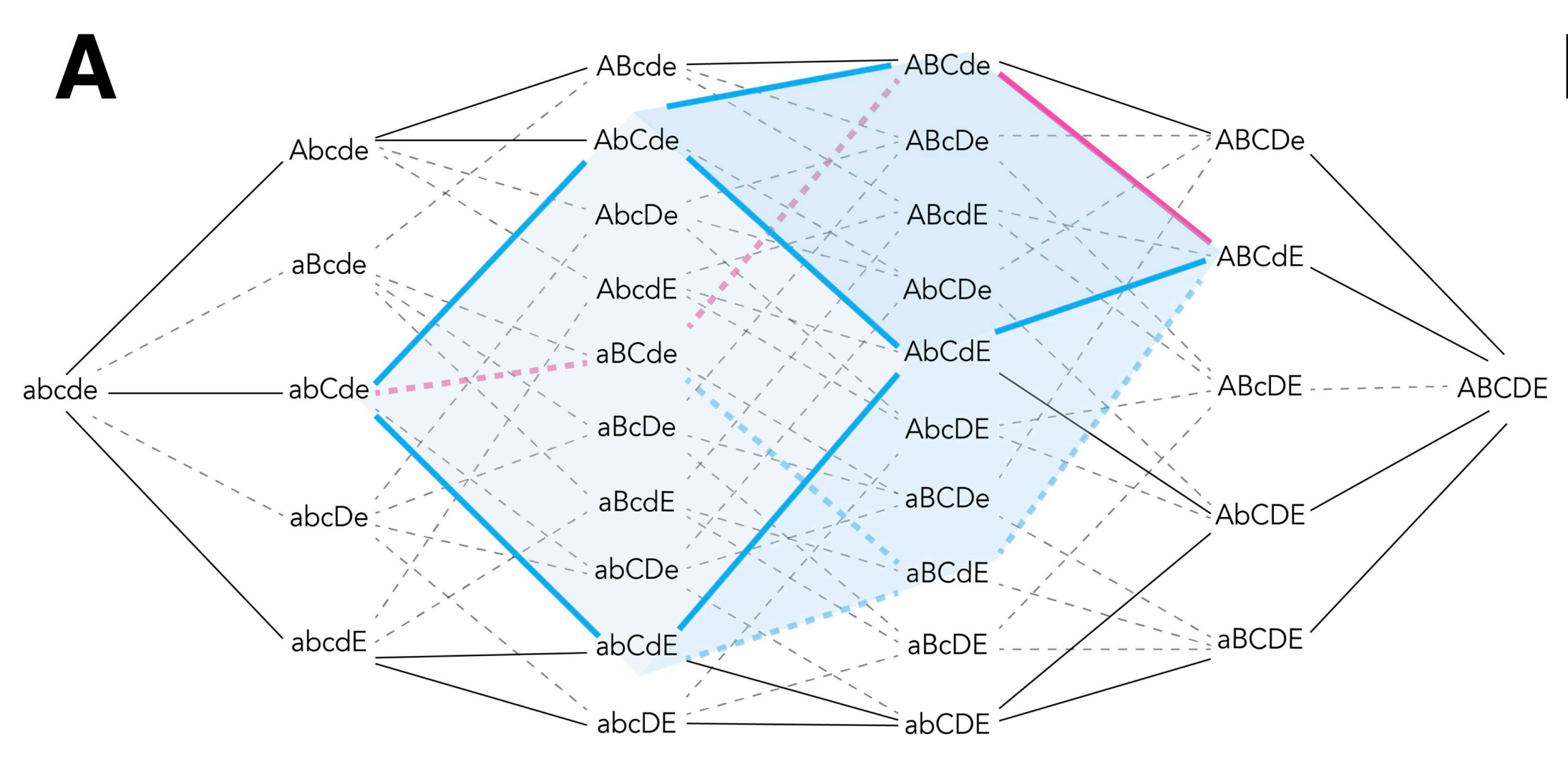

B

C

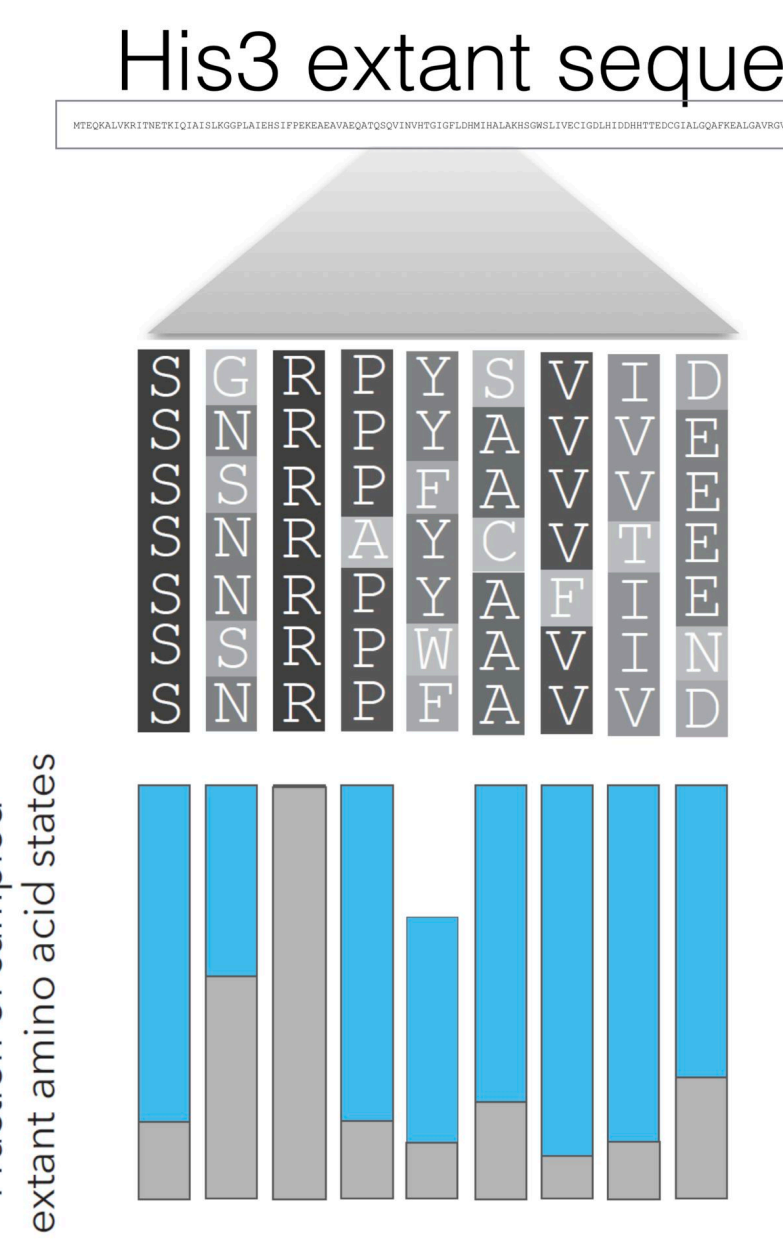

D

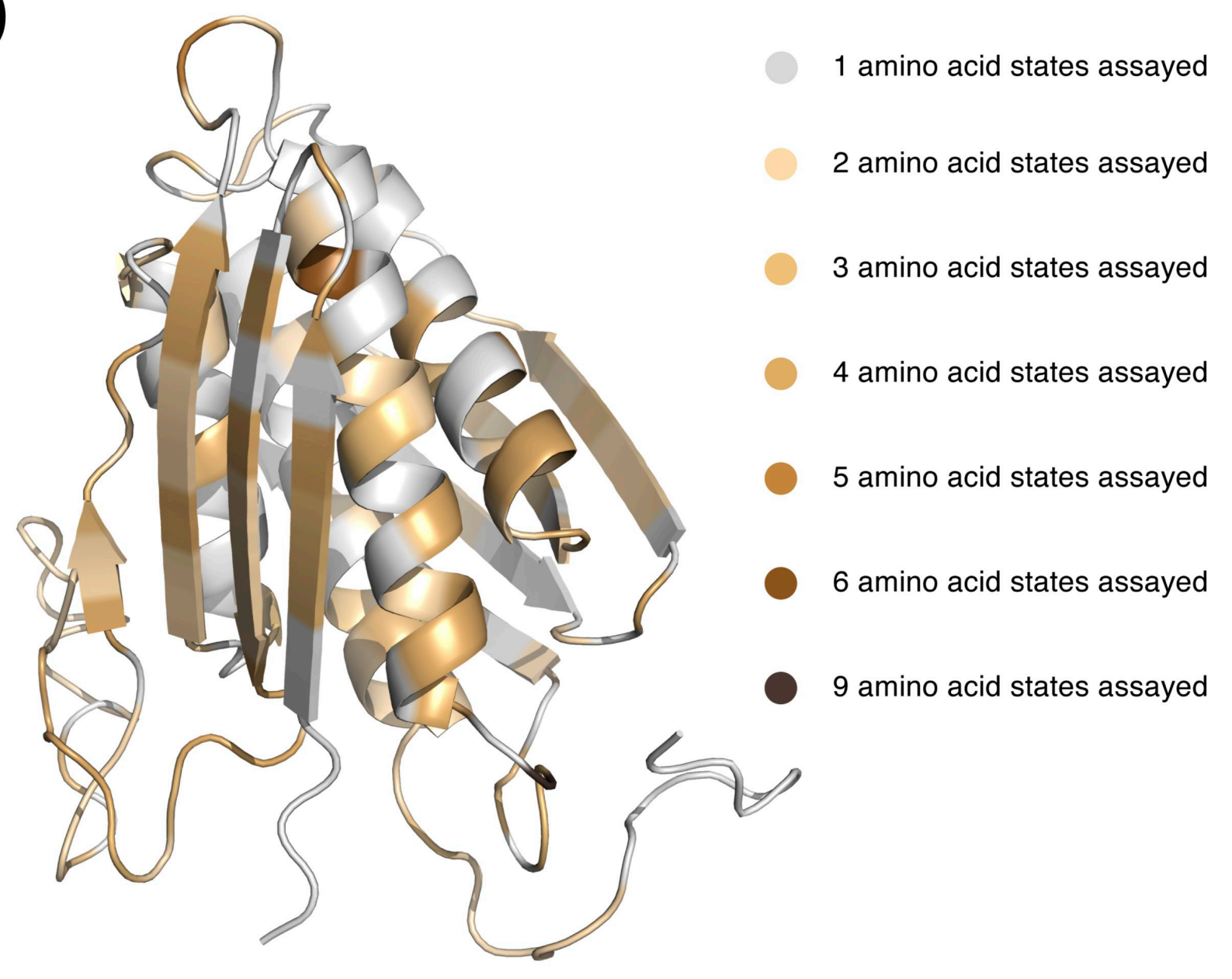




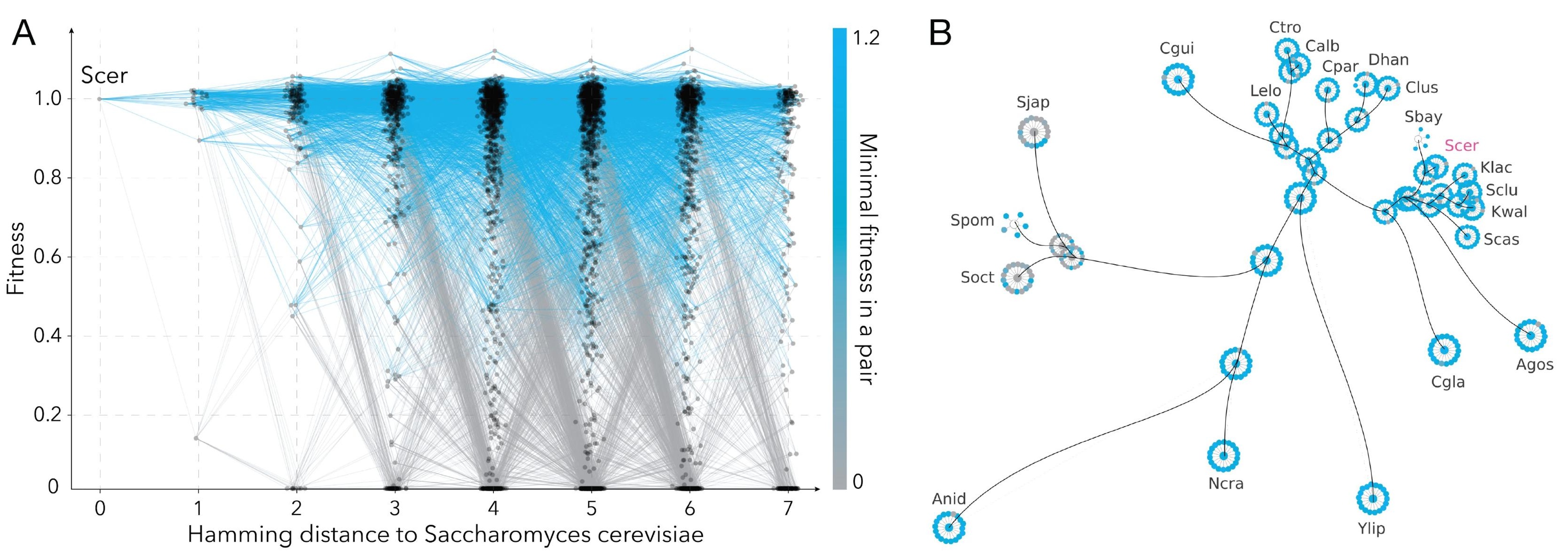




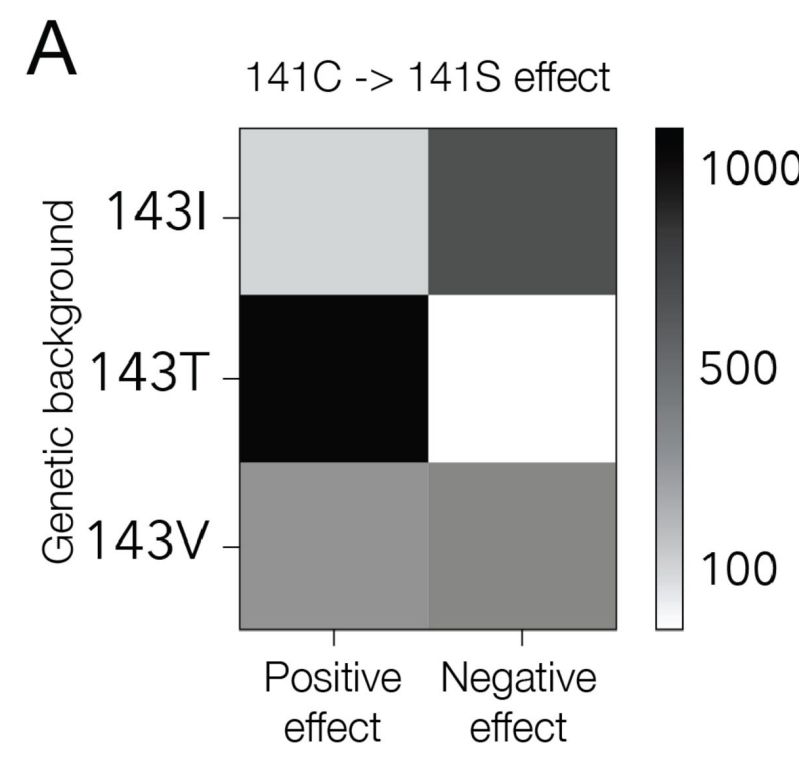

C
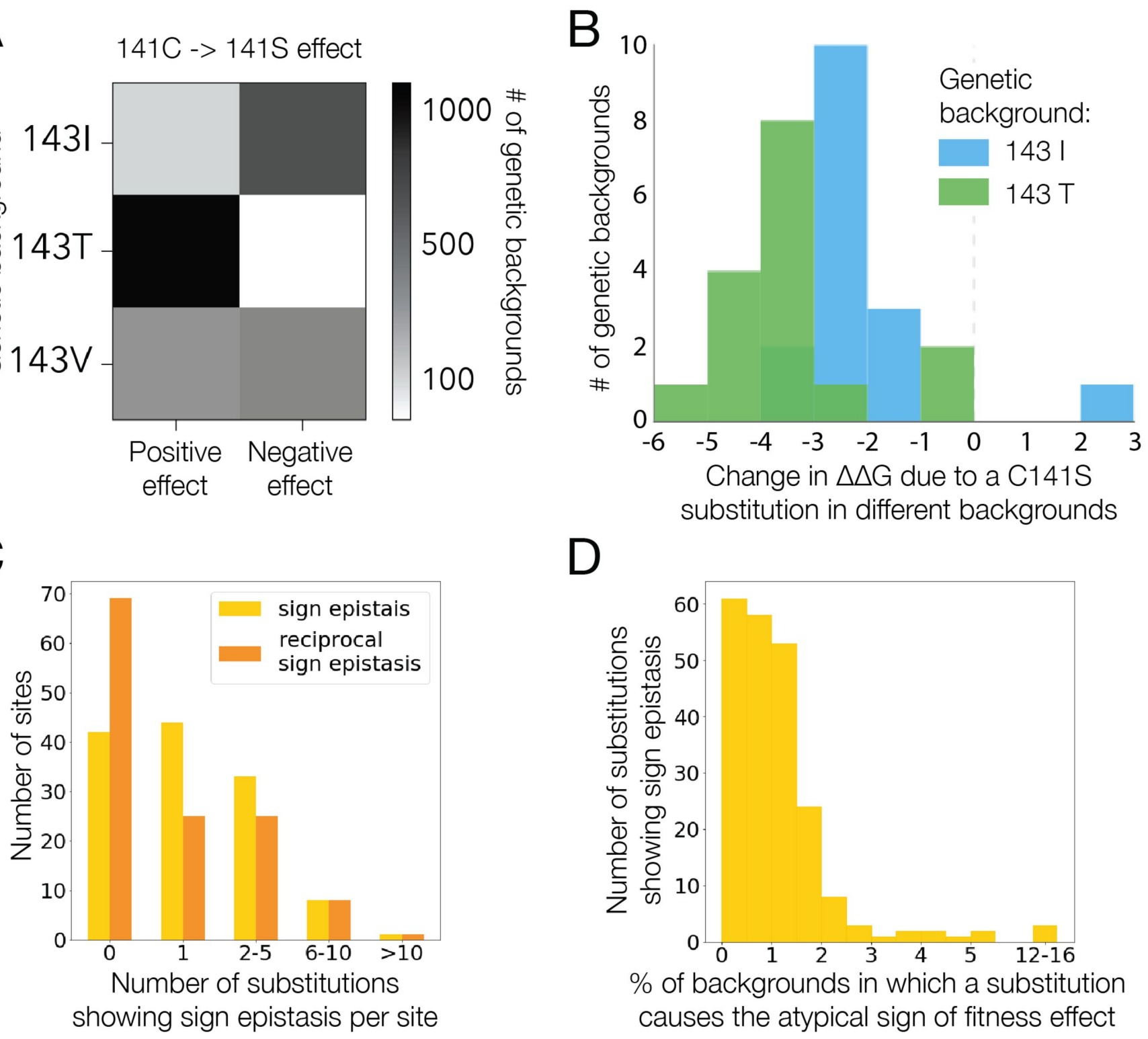

D

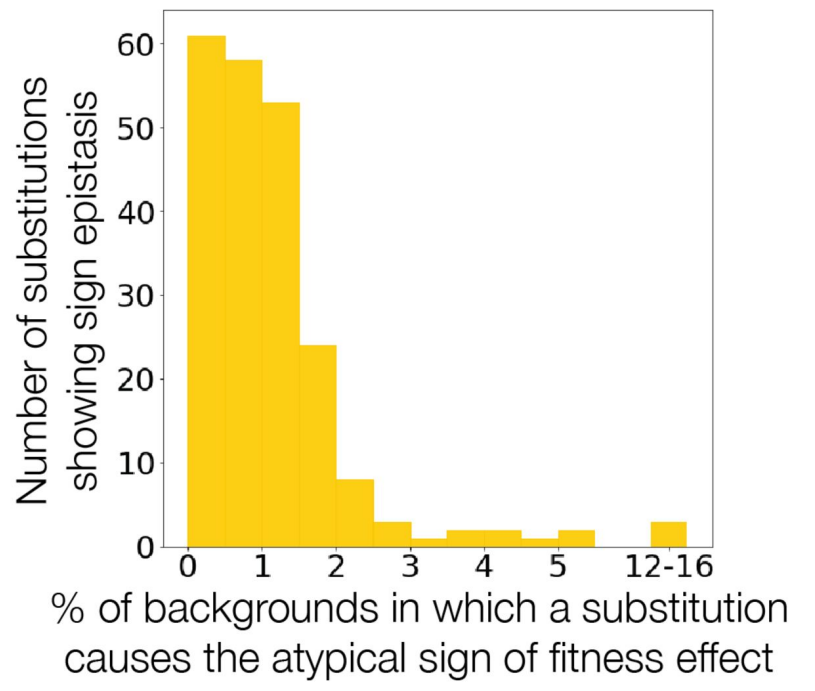




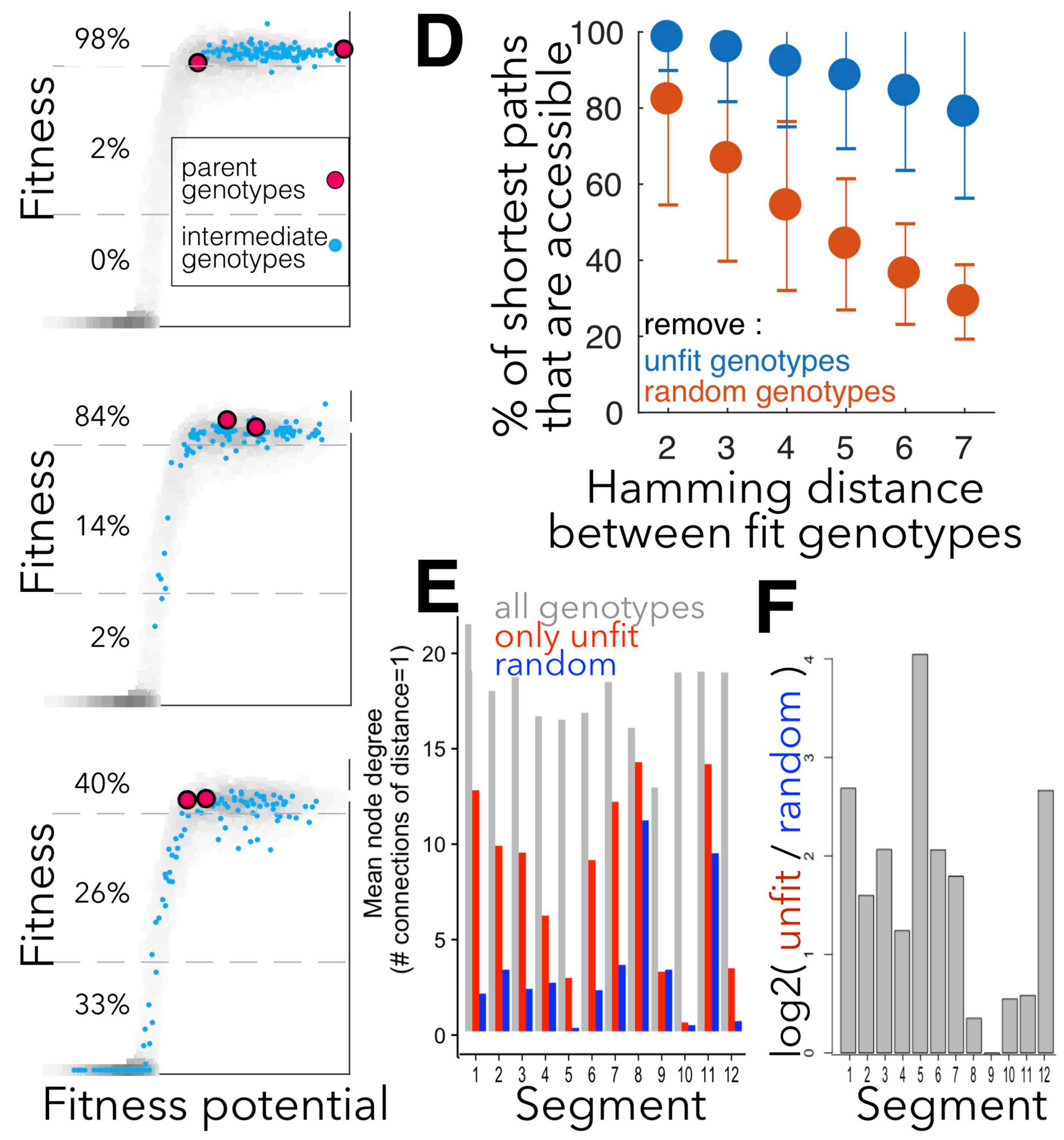




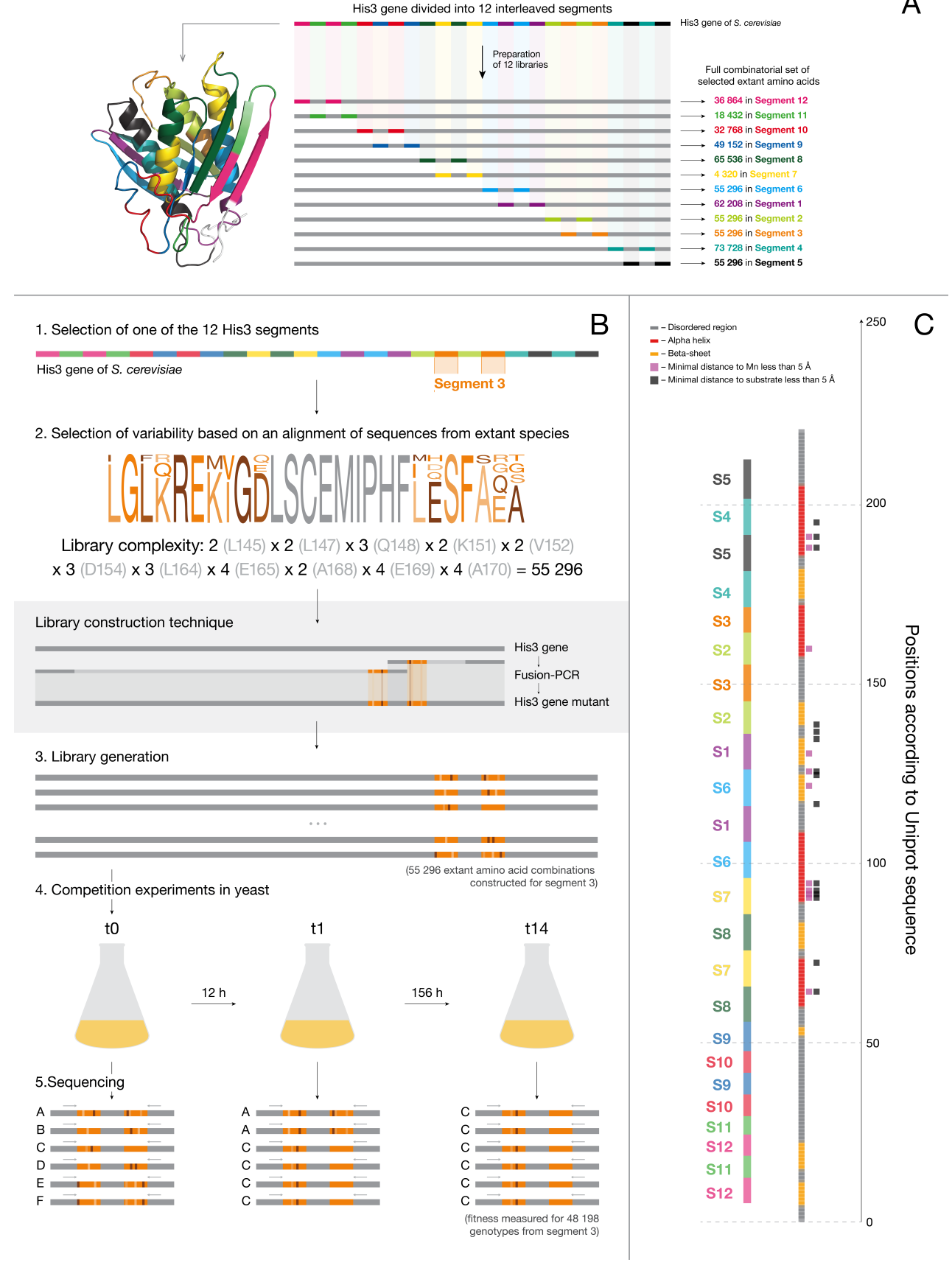


A

Extant Non-Extant, same position
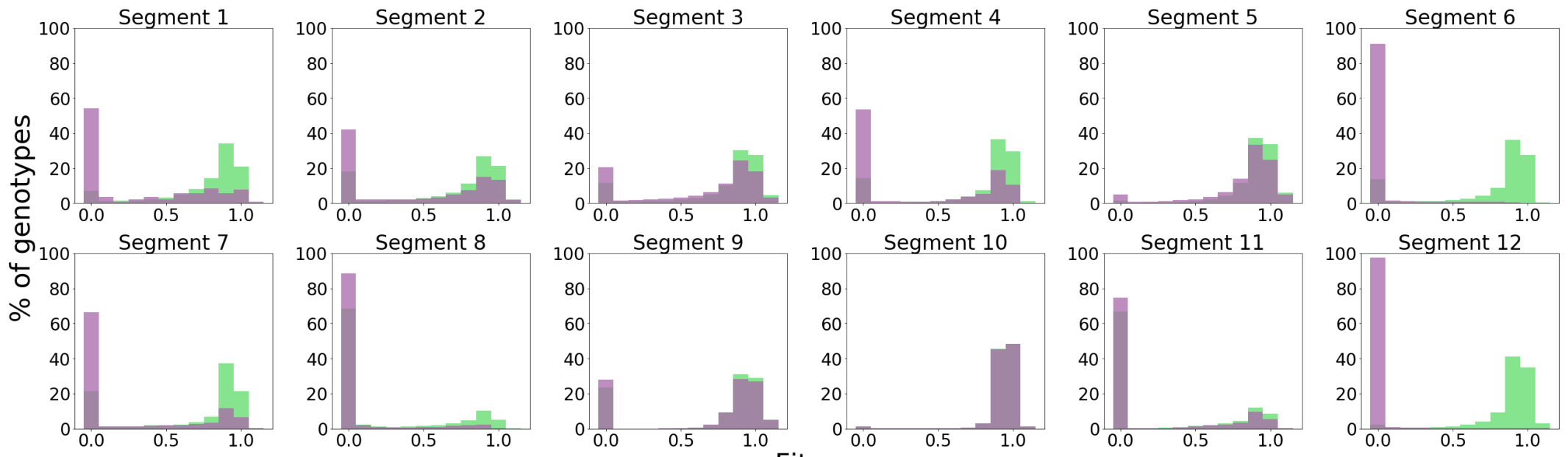

B

\section{Fitness}

\section{Extant Non-Extant, different position}
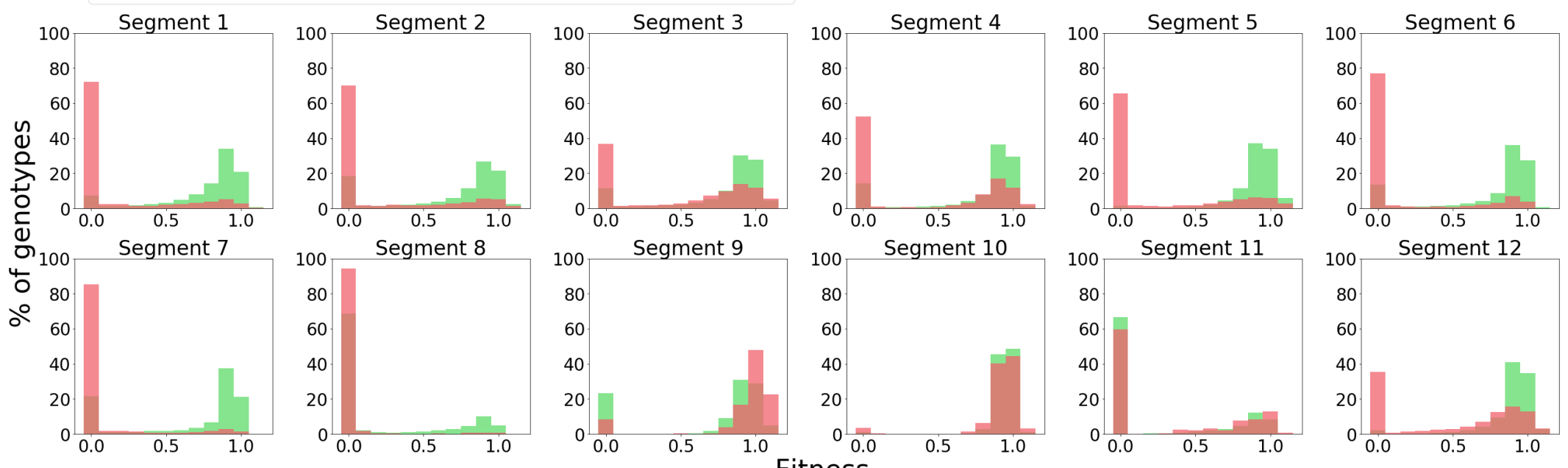

Fitness 
A
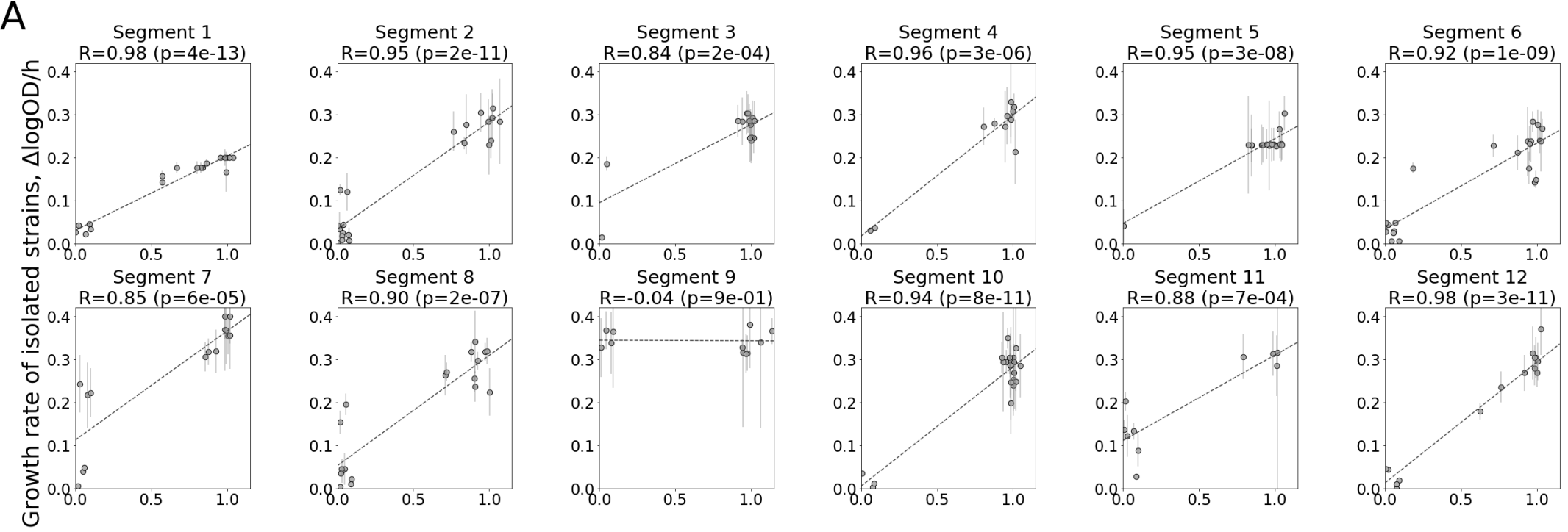

Fitness from competition assay

\section{B}

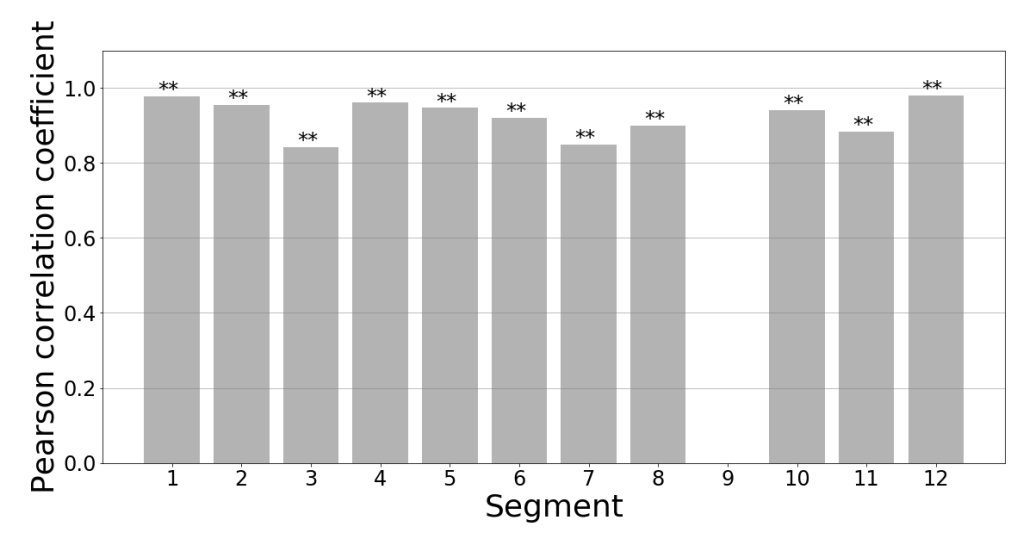

C

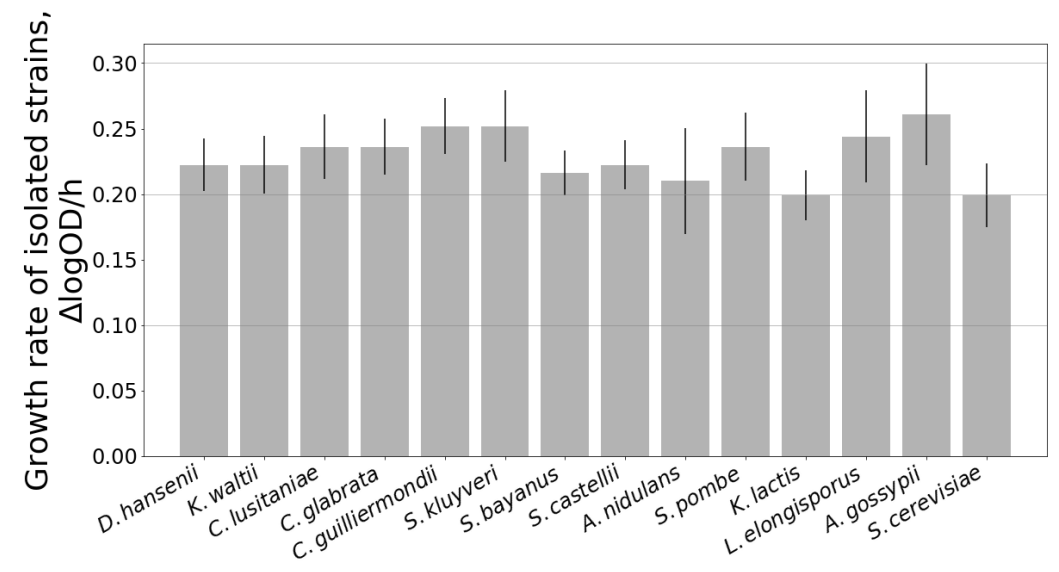




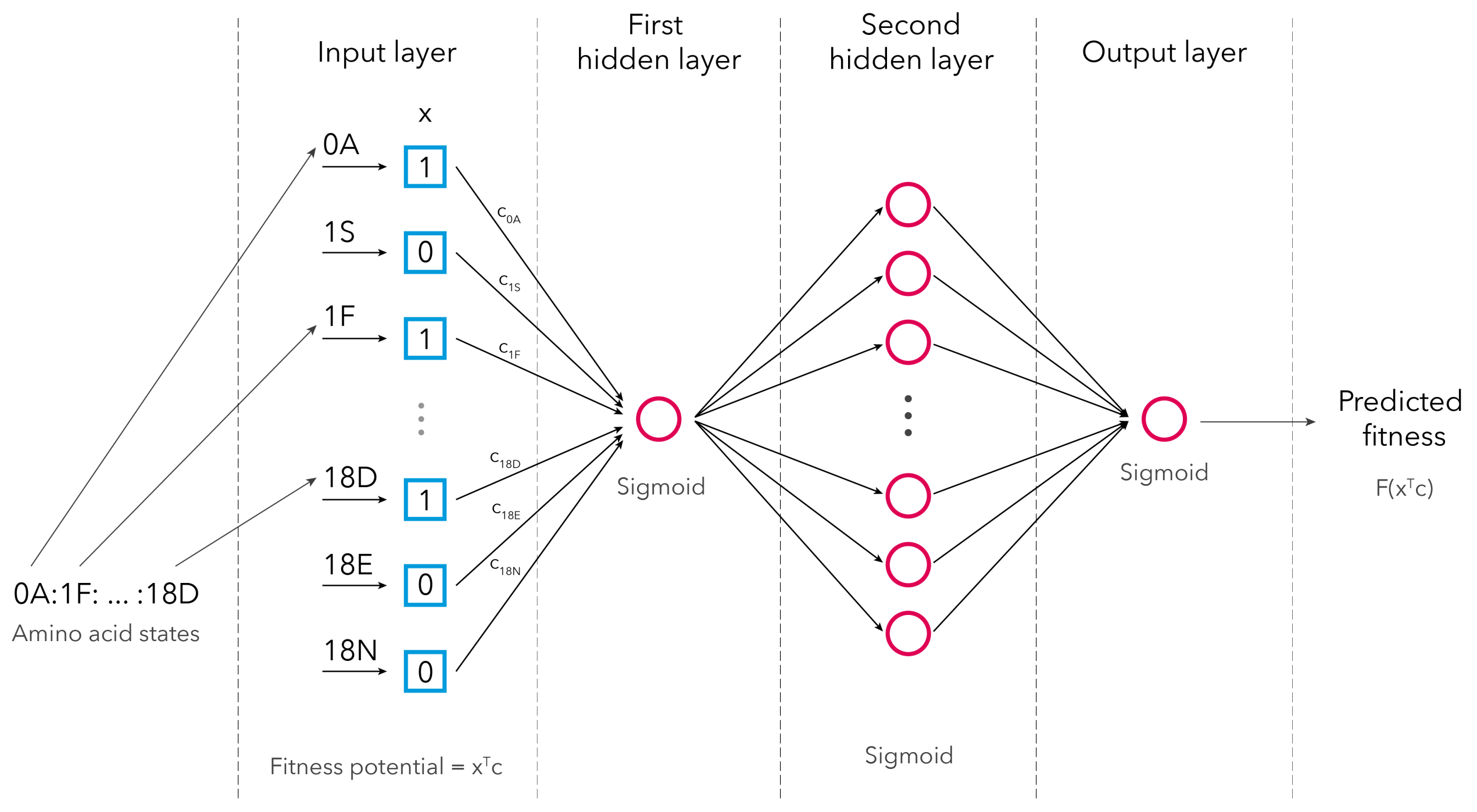


A
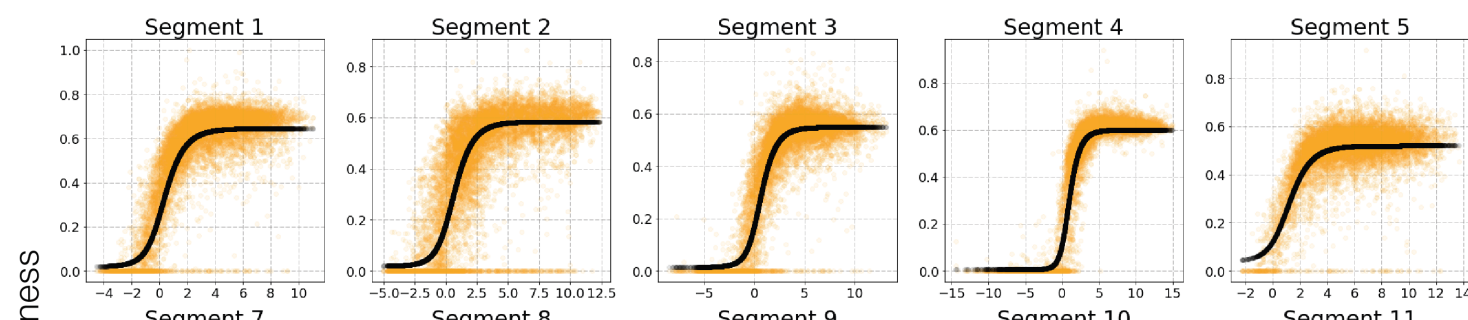

Segment 6

1.0 Segment 7
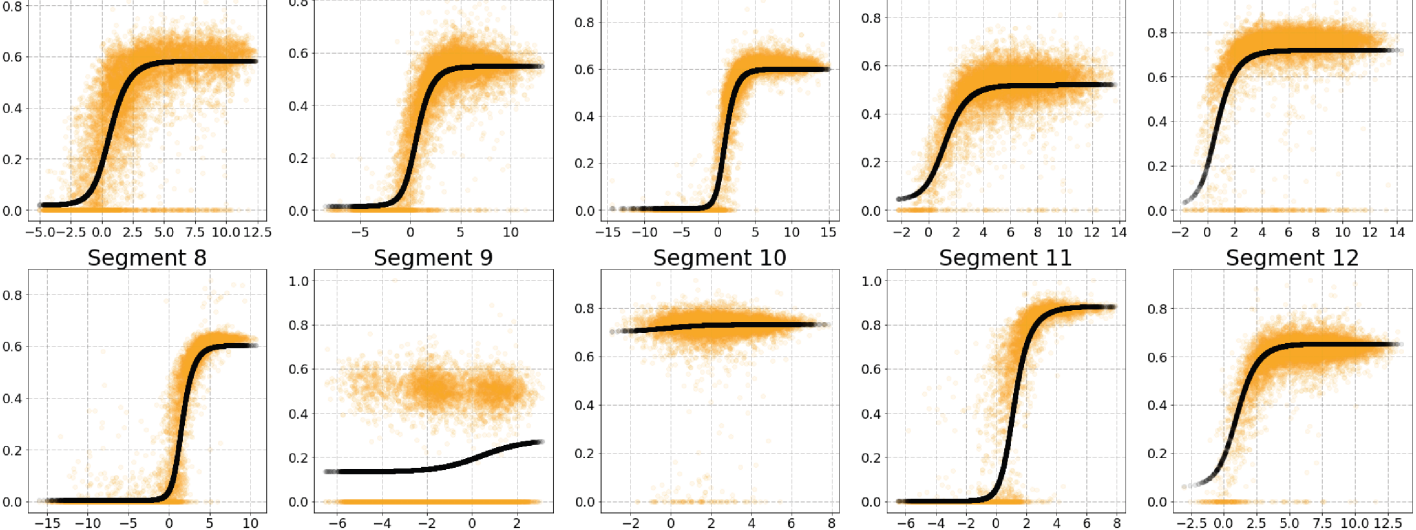

\section{B}
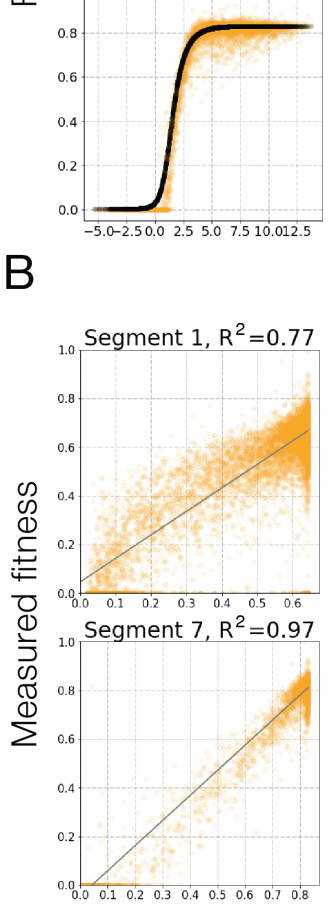

C
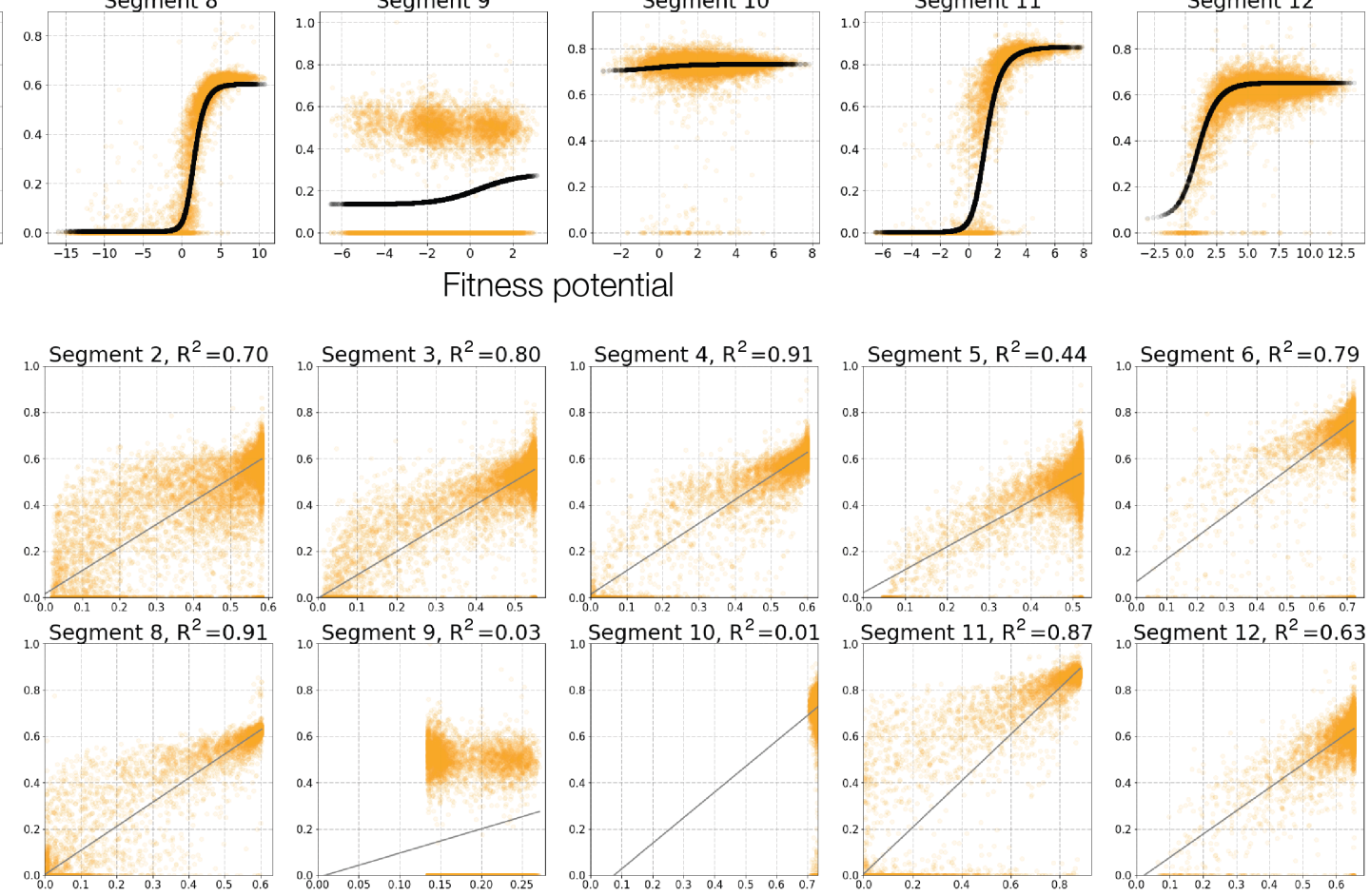

Fitness potentia

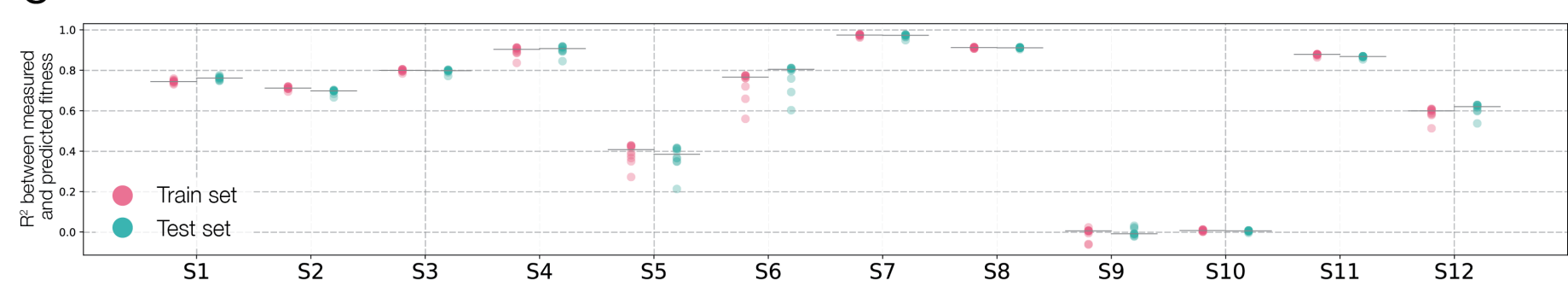
Predicted fitness 

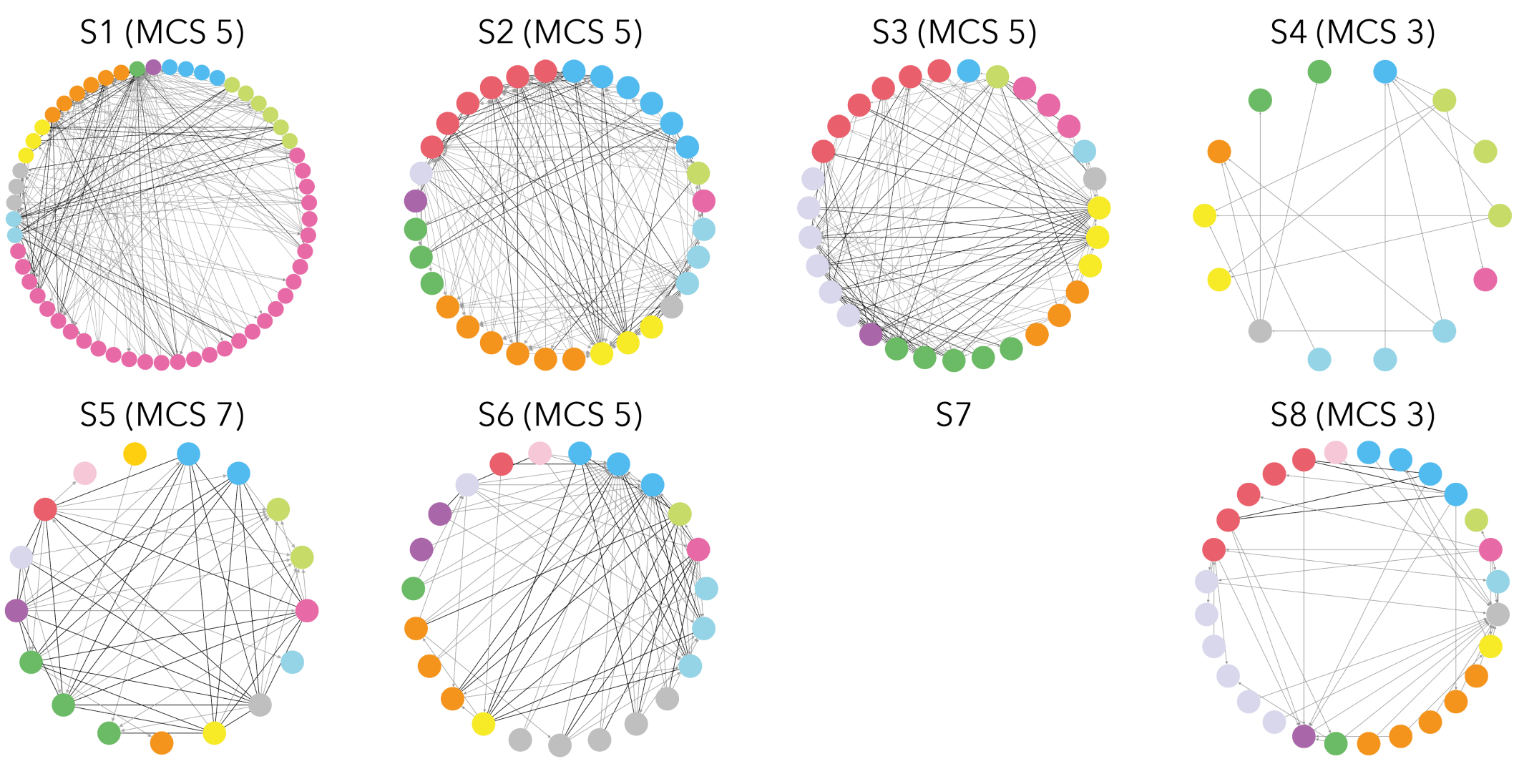

S7
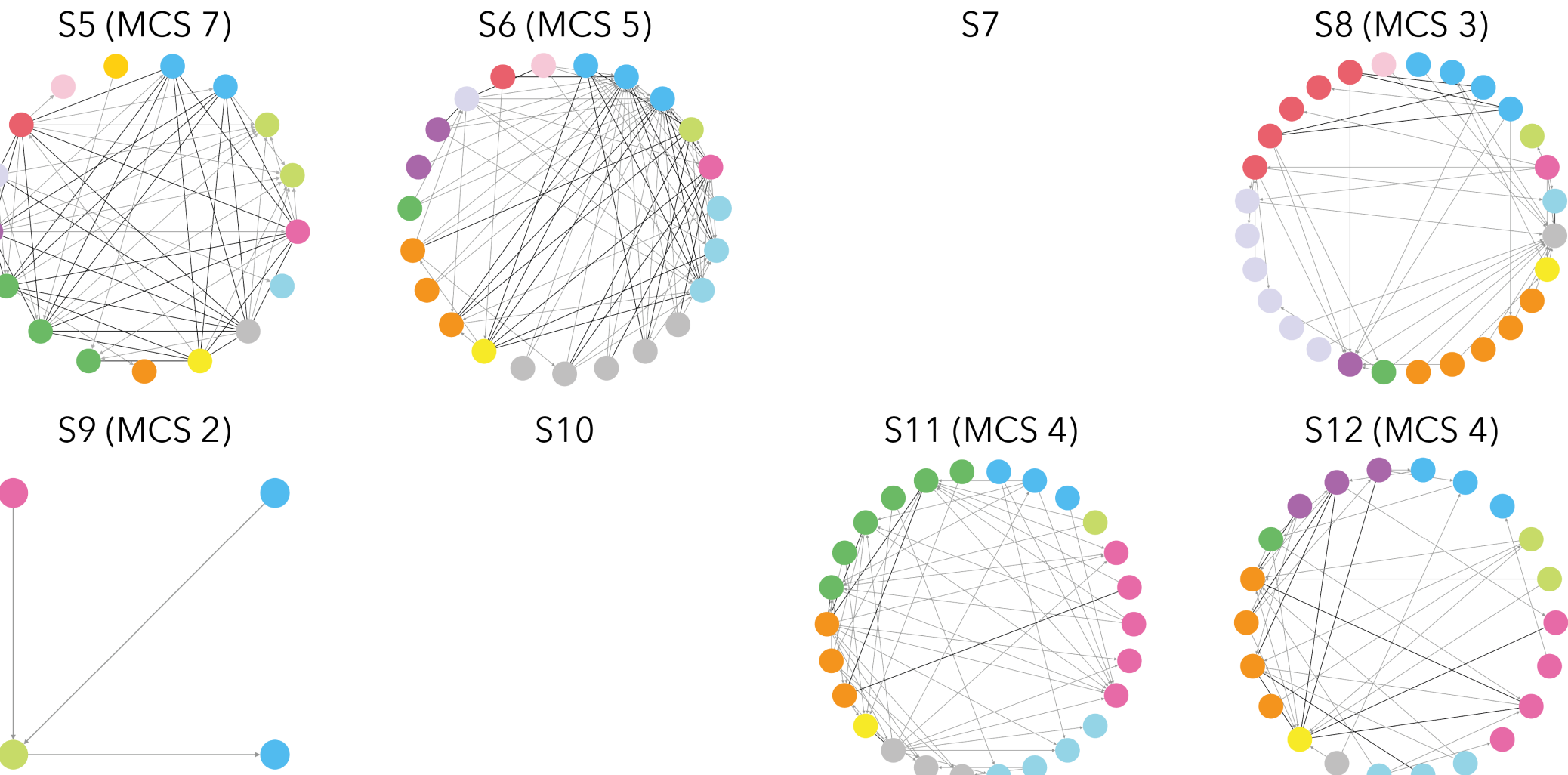

S10
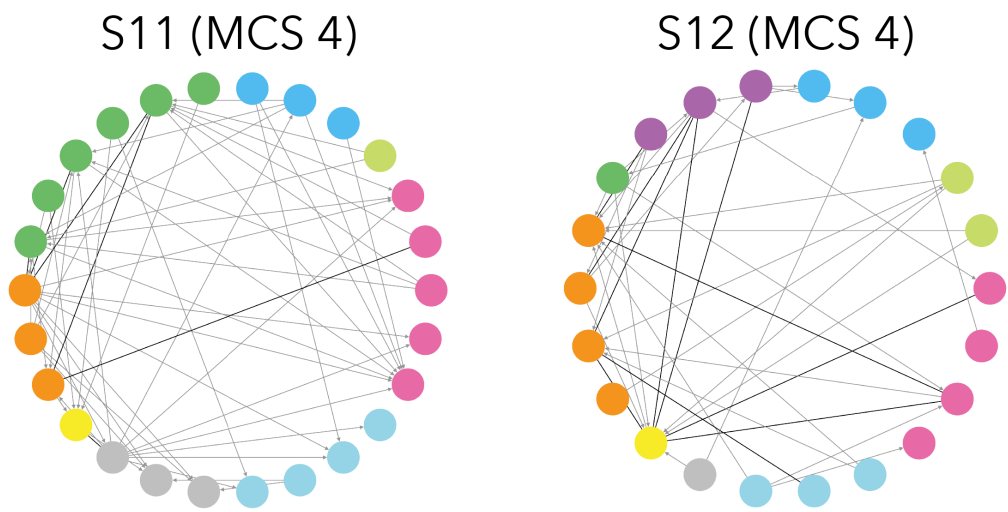
A
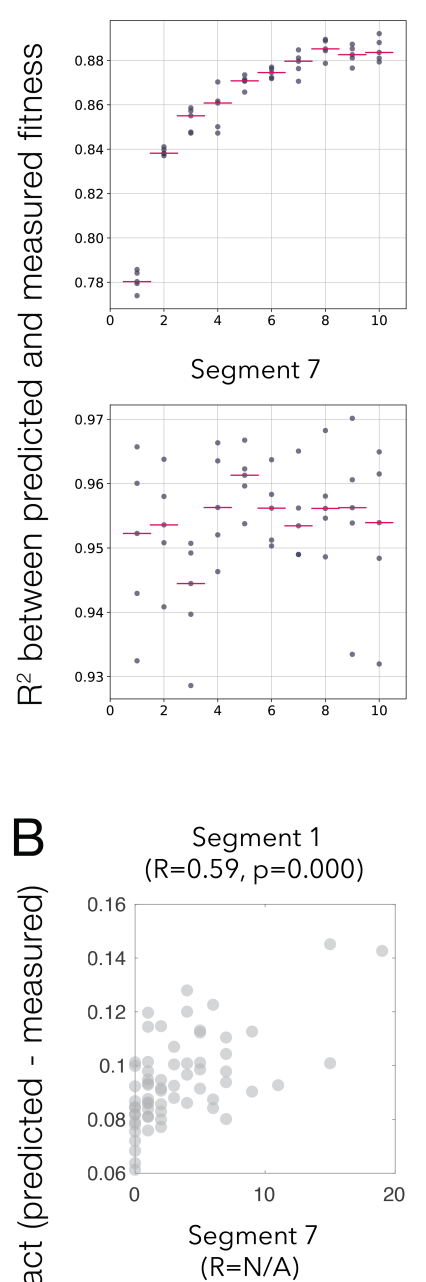

है
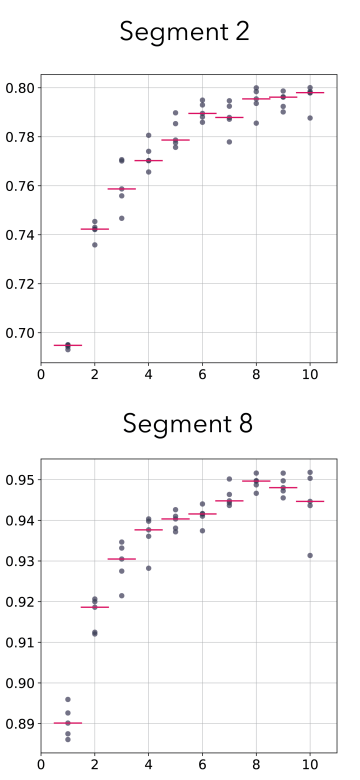

Segment 3

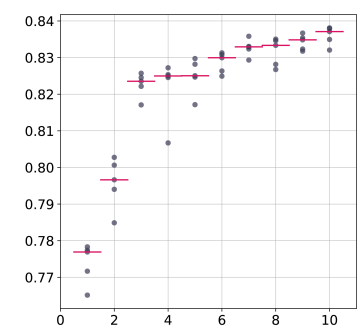

Segment 9

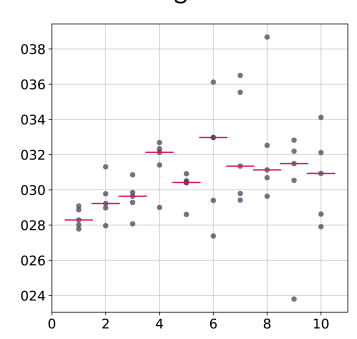

Segment 4

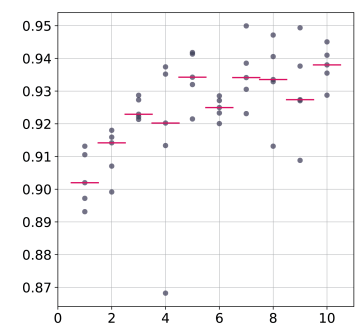

Segment 10

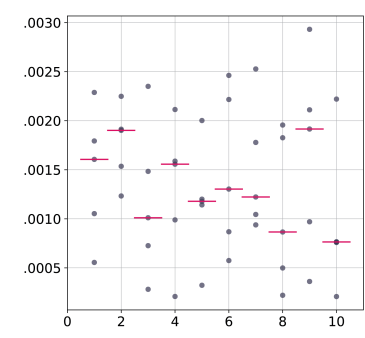

Segment 5

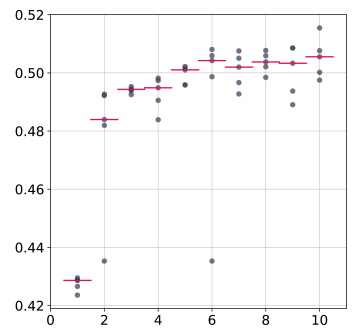

Segment 11

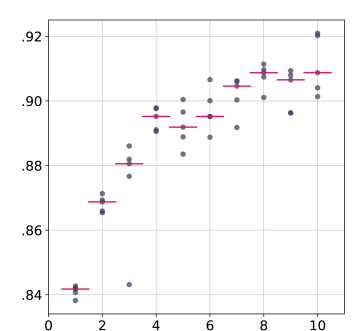

Segment 6

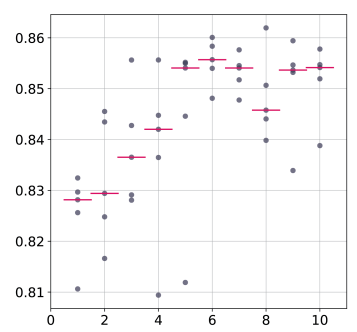

Segment 12

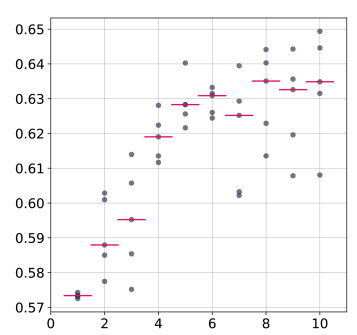

Number of neurons in the first layer of the neural network architecture
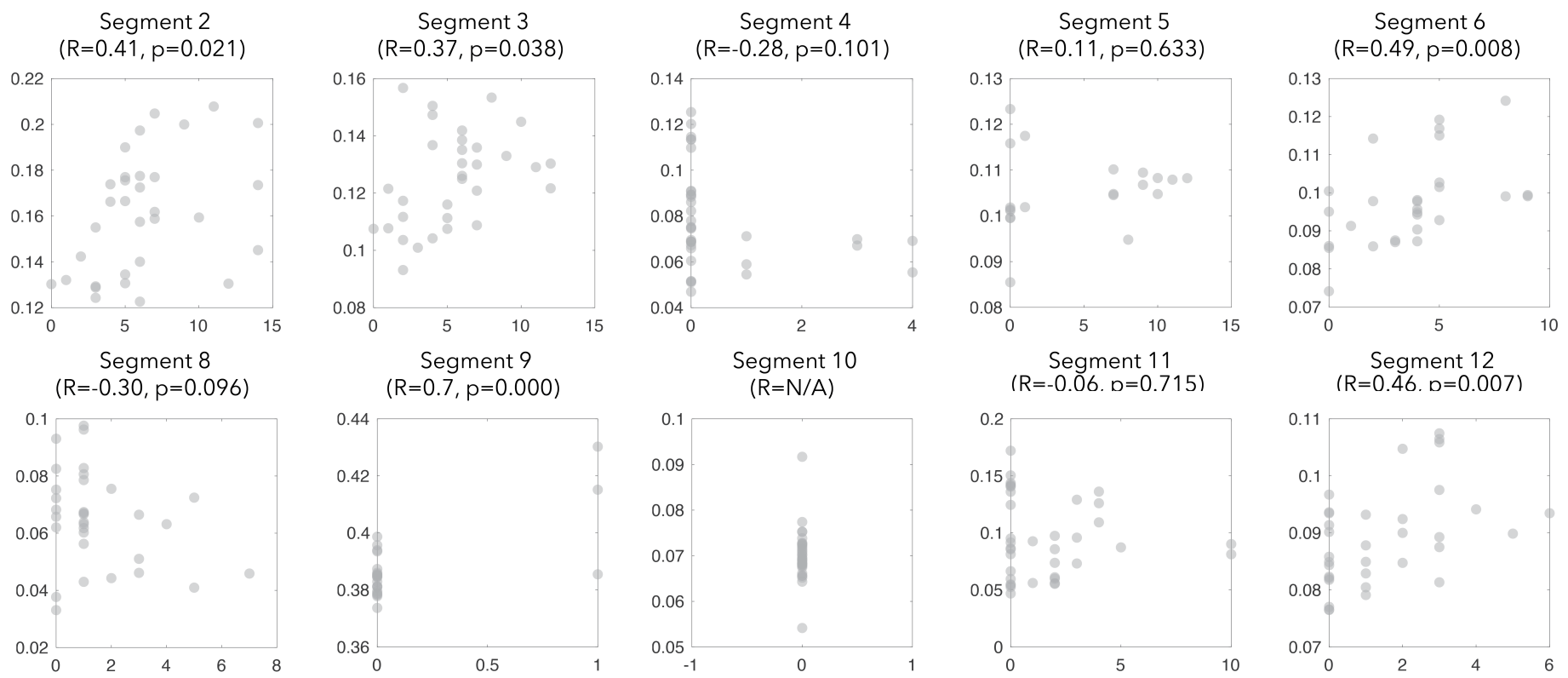

Number of interactions for each substitution with sign epistasis 


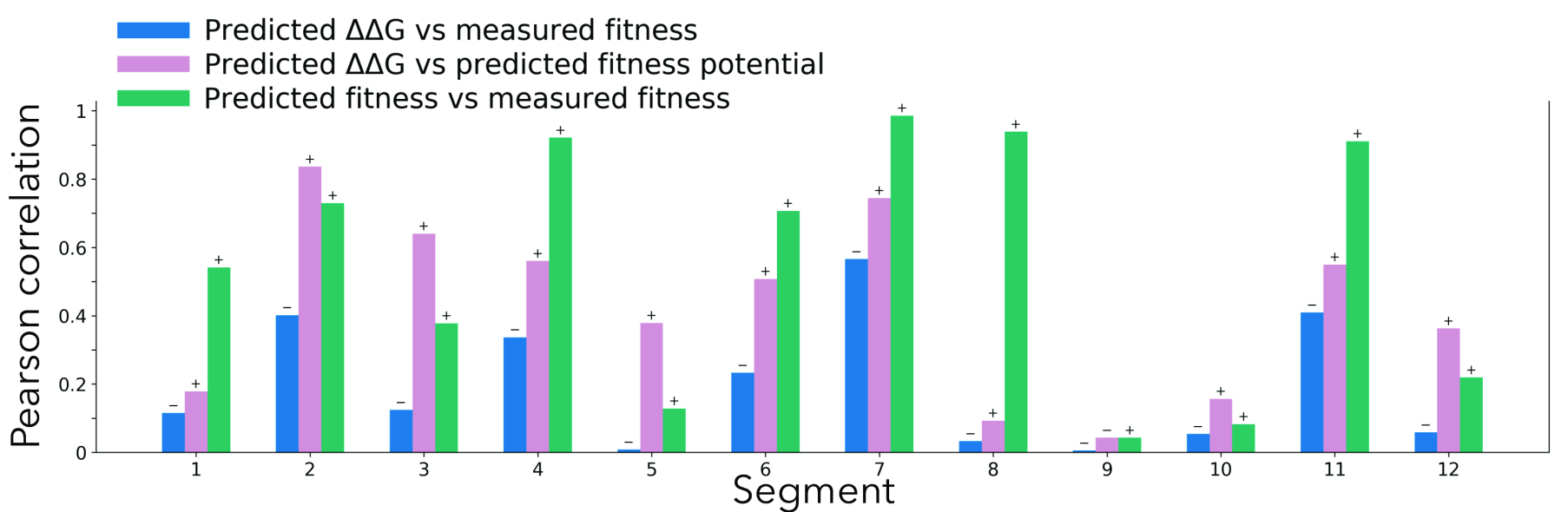

B

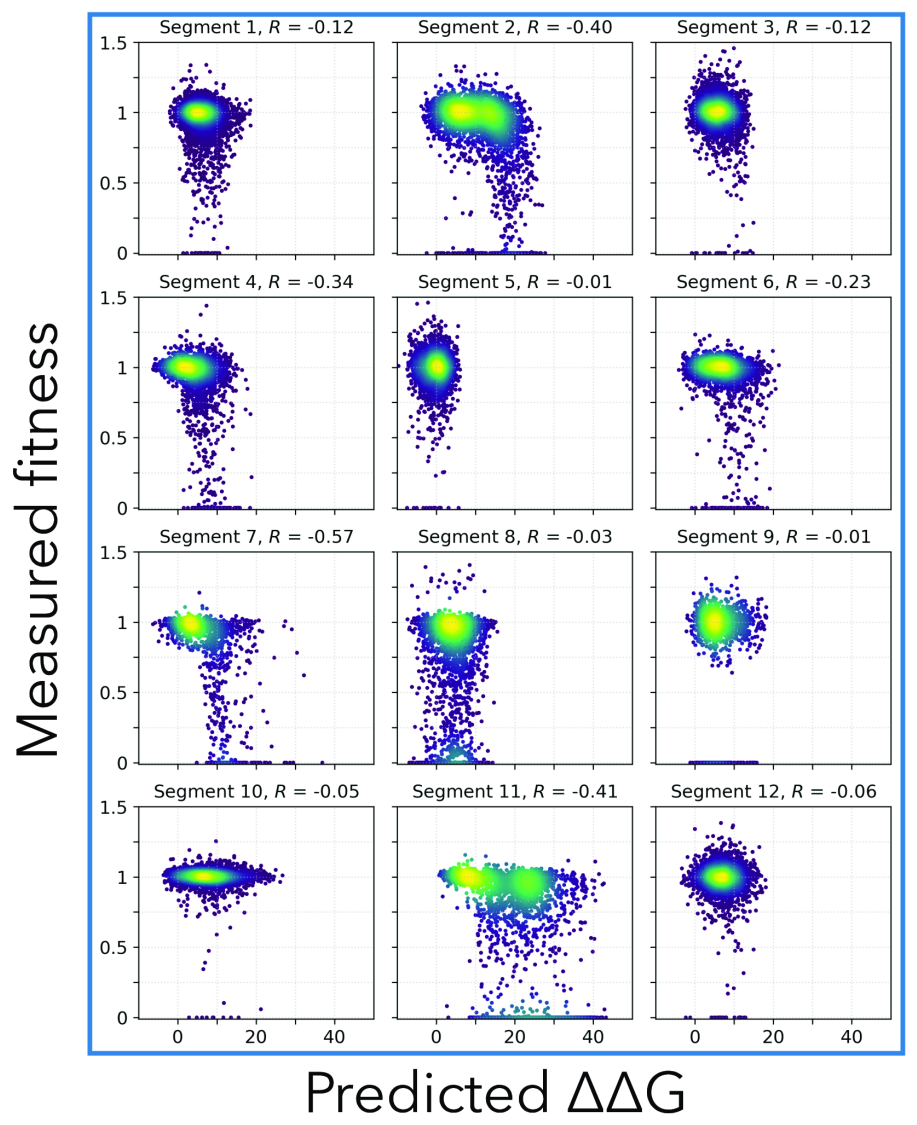

C

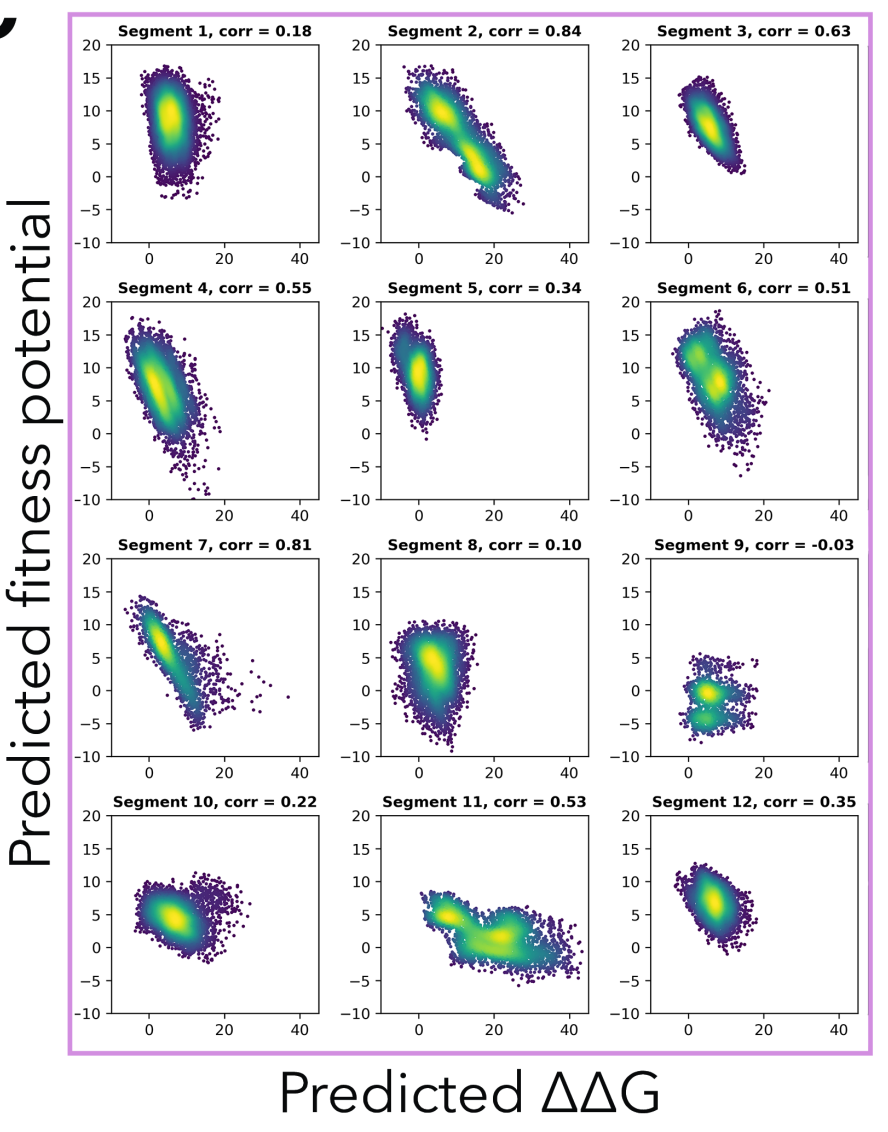

D

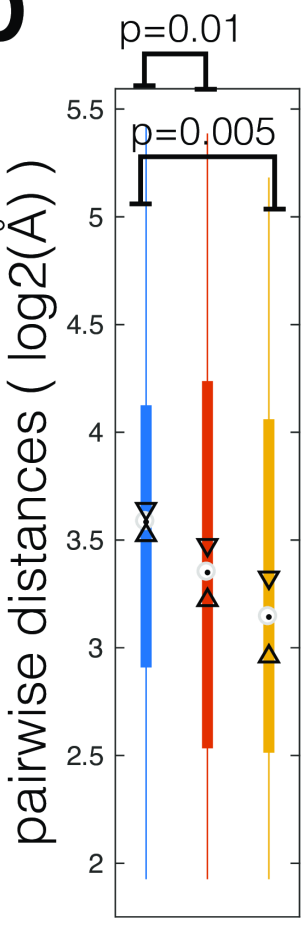

$\overline{0} \frac{\bar{\alpha}}{0} \frac{\bar{\alpha}}{\mathrm{d}}$ $ᄃ ᄃ$

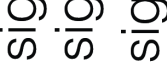
$\circ \bar{\sigma}$

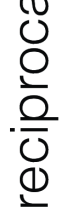


A

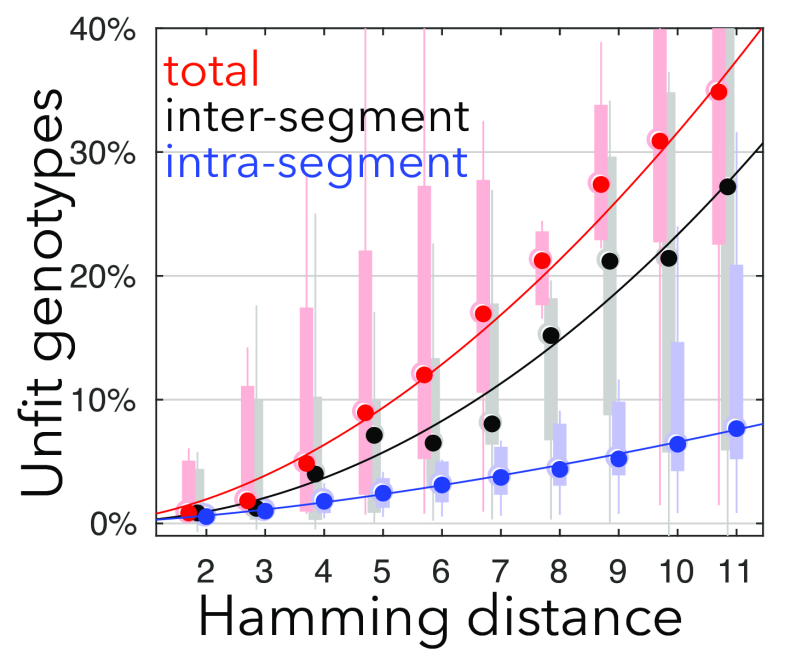

B

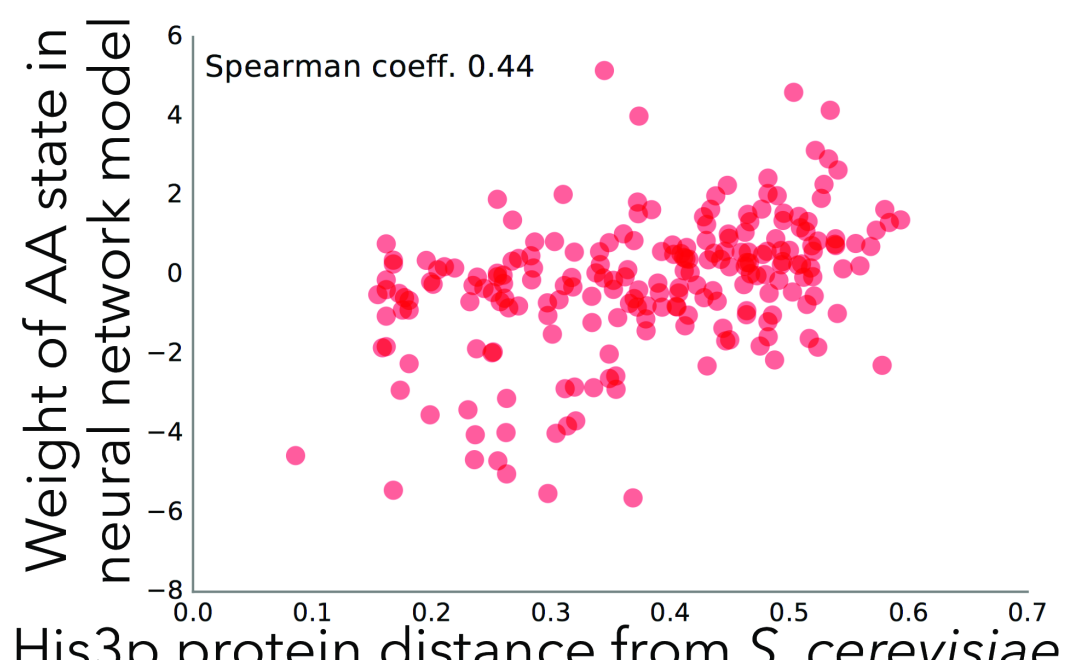
His3p protein distance from S. cerevisiae to that amino acid state 